\title{
The Minimum and Other Free Energies for Non-Linear Materials With Memory.
}

John Murrough Golden

Technological University Dublin, Murrough.Golden@TUDublin.ie

Follow this and additional works at: https://arrow.tudublin.ie/scschmatart

Part of the Mathematics Commons

\section{Recommended Citation}

Golden, M. (2015) The Minimum and Other Free Energies for Non-Linear Materials With Memory. "Quarterly of applied mathematics" Vol. 74, pg. 137-164. American Mathematical Society. Published electronically: December 4, 2015. doi:10.1090/qam/1407 http://dx.doi.org/10.1090/qam/1407

This Article is brought to you for free and open access by the School of Mathematics at ARROW@TU Dublin. It has been accepted for inclusion in Articles by an authorized administrator of ARROW@TU Dublin. For more information, please contact arrow.admin@tudublin.ie, aisling.coyne@tudublin.ie,gerard.connolly@tudublin.ie.

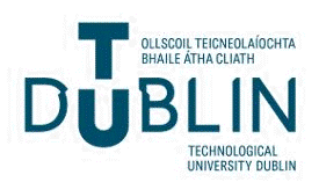




\title{
THE MINIMUM AND OTHER FREE ENERGIES FOR NON-LINEAR MATERIALS WITH MEMORY
}

\author{
BY \\ J. M. GOLDEN
}

School of Mathematical Sciences, Dublin Institute of Technology, Kevin Street, Dublin 8, Ireland

\begin{abstract}
Expressions are obtained for free energies of materials with a certain type of non-linear constitutive relation. In particular, the minimum and related free energies are considered in some detail. Minimal states are defined for these materials, and it is shown that any free energy yielding a linear constitutive equation that is a functional of the minimal state has a counterpart in the non-linear case which is also a minimal state functional in this more general context. These results are explored for simple examples, including discrete spectrum materials.
\end{abstract}

1. Introduction. There are generally many free energies associated with a material with memory. They form a bounded convex set with a minimum and a maximum element (10], for example). Explicit expressions for the minimum free energy and various related free energy functionals, in the case of materials with constitutive equations involving only linear memory terms, are derived in a series of papers over more than a decade, starting with [12. Much of this work is summarized in [2, which also discusses other free energy functionals yielding such constitutive equations.

In the present work, we generalize these results to materials with non-linear constitutive equations of a particular type.

On the matter of notation, a group of relations with a single equation number $(* * *)$ will be distinguished by counting "=" signs. Thus, $(* * *)_{5}$ refers to the relation with the fifth "=" sign.

We will be considering frequency domain quantities, defined by analytic continuation from integral definitions, as functions on the complex $\omega$ plane $\Omega$, where

$$
\begin{aligned}
\Omega^{+} & =\left\{\omega \in \Omega \mid I m \omega \in \mathbb{R}^{+}\right\}, \\
\Omega^{(+)} & =\left\{\omega \in \Omega \mid I m \omega \in \mathbb{R}^{++}\right\} .
\end{aligned}
$$

Received June 16, 2014.

2010 Mathematics Subject Classification. Primary 80A17, 74D10.

Key words and phrases. Thermodynamics, memory effects, free energy functional, non-linear constitutive equation, rate of dissipation.

E-mail address: murrough.golden@dit.ie 
Similarly, $\Omega^{-}$and $\Omega^{(-)}$are the lower half-planes including and excluding the real axis, respectively.

2. Non-linear models of materials with memory. In this section, we discuss general aspects of non-linear (and linear) theories of materials with memory. The discussion is presented for the scalar version of the theory in order to describe developments in the simplest terms. However, it is a straightforward matter to generalize the arguments to a finite vector space $\Gamma$, which may be $S y m$ if the material is viscoelastic under isothermal conditions, or $\mathbb{R}^{3} \times \mathbb{R}$ for heat conductors, or combinations of these. Theories of dielectrics or electromagnetic conductors could also be fitted into such a scheme, with minor modifications.

Our independent variable at time $t$ and its history are $E(t)$ and $E^{t}$, where

$$
E^{t}(s)=E(t-s), \quad s \in \mathbb{R}^{+},
$$

while the relative history is given by

$$
E_{r}^{t}(s)=E^{t}(s)-E(t), \quad s \in \mathbb{R}^{+} .
$$

The dependent variable is denoted by $S(t)$. The general form of the constitutive relation is

$$
S(t)=\widetilde{S}\left(E^{t}, E(t)\right),
$$

where it is understood that $\widetilde{S}$ is a functional of $E^{t}$ and a function of $E(t)$. We shall, for convenience, often refer to $S(t)$ as the stress and $E(t)$ as the strain. The general form of a free energy functional is denoted by

$$
\psi(t)=\widetilde{\psi}\left(E^{t}, E(t)\right) \geq 0,
$$

where, as with $\widetilde{S}$, the quantity $\widetilde{\psi}$ is a functional of $E^{t}$ and a function of $E(t)$. Let $E^{\dagger}$ be the static history, equal to $E(t)$ at the current and all past times. Then

$$
\widetilde{\psi}\left(E^{\dagger}, E(t)\right)=\widetilde{\phi}(E(t)) \geq 0,
$$

where $\widetilde{\phi}(E(t))$ is the equilibrium free energy. This is a definition of $\widetilde{\phi}$, which must have the same form for any choice of free energy. The notation

$$
\phi(t)=\widetilde{\phi}(E(t))
$$

will be used frequently.

2.1. Required properties of a free energy. Let us state the properties a functional must have if it is to be a free energy.

P1: We have

$$
\frac{\partial \widetilde{\psi}\left(E^{t}, E(t)\right)}{\partial E(t)}=\frac{\partial \psi(t)}{\partial E(t)}=S(t)=\widetilde{S}\left(E^{t}, E(t)\right) .
$$

Thus, any choice of free energy must yield the same $\widetilde{S}$, according to this prescription.

P2: For any history and current value $\left(E^{t}, E(t)\right)$,

$$
\widetilde{\psi}\left(E^{t}, E(t)\right) \geq \widetilde{\phi}(E(t)),
$$

where equality is achieved only for the static history $E^{\dagger}$. 
P3: We have

$$
\dot{\psi}(t)+D(t)=S(t) \dot{E}(t), \quad D(t) \geq 0,
$$

where $D(t)$ is the rate of energy dissipation. The first relation is a statement of the first law, while the non-negativity of $D(t)$ is in effect the second law.

These are a version of the Graffi conditions for a free energy [17. Note that properties P1 and P2 follow from the second law, which is included in P3 3]. We shall discuss later a particular example of how P2 is a consequence of the non-negative property of $D(t)$.

Let us assume that $\lim _{t \rightarrow-\infty} \psi(t)=0$. Then, integrating (2.9) over all past history, we obtain

$$
\psi(t)+\mathcal{D}(t)=W(t)
$$

where $\mathcal{D}(t)$ is the total dissipation in the material up to time $t$, defined by

$$
\mathcal{D}(t)=\widetilde{\mathcal{D}}\left(E^{t}, E(t)\right)=\int_{-\infty}^{t} D(u) d u \geq 0, \quad \dot{\mathcal{D}}(t)=D(t),
$$

while $W(t)$ is the work function, given by

$$
W(t)=\int_{-\infty}^{t} S(u) \dot{E}(u) d u=\widetilde{W}\left(E^{t}, E(t)\right)=W(t ; E(t)) .
$$

The last notation will be used later. It is assumed that the integrals in (2.11) and (2.12) exist.

REMARK 2.1. We note that $W(t)$ behaves similarly to a free energy functional with zero dissipation associated with it. From (2.12), it follows that

$$
\dot{W}(t)=S(t) \dot{E}(t),
$$

which is (2.9) or P3 for $D(t)=0$. It is also clear from (2.8) and (2.10) that

$$
W(t) \geq \psi(t) \geq \phi(t)
$$

where $\psi(t)$ is any free energy functional, so that $W(t)$ is either the maximum free energy or greater than this quantity.

Let us now briefly demonstrate that it also obeys P1, using an intuitive and somewhat modified version of the argument in [3], and P2. Relation (2.14) is equivalent to (2.8), though it must be shown that equality is achieved only for static histories. We can write

$$
\dot{W}(t)=\frac{\partial}{\partial E(t)} \widetilde{W}\left(E^{t}, E(t)\right) \dot{E}(t)+\delta \widetilde{W}
$$

where the rightmost term is a Fréchet differential of $\widetilde{W}$, defined within a suitable Hilbert space (for example [2], page 112). Thus, (2.13) can be written in the form

$$
\left[\frac{\partial}{\partial E(t)} \widetilde{W}\left(E^{t}, E(t)\right)-\widetilde{S}\left(E^{t}, E(t)\right)\right] \dot{E}(t)=-\delta \widetilde{W} .
$$

The quantity $\dot{E}(t)$ can take arbitrary values, so that (2.7) or P1 must hold for $\widetilde{\psi}$ replaced by $\widetilde{W}$, giving

$$
\frac{\partial}{\partial E(t)} W(t)=S(t)
$$


Also, the quantity $\delta \widetilde{W}$ must vanish. From (2.7) for both $\widetilde{\psi}$ and $\widetilde{W}$, together with (2.10), it follows that

$$
\frac{\partial \mathcal{D}(t)}{\partial E(t)}=0
$$

giving

$$
\widetilde{\mathcal{D}}\left(E^{t}, E(t)\right)=\widetilde{\mathcal{D}}\left(E^{t}\right) .
$$

Using (2.19) and (2.10), we obtain

$$
\frac{\partial}{\partial E(t)} \psi(t)=\frac{\partial}{\partial E(t)} W(t)=S(t)
$$

which explicitly provides a unique constitutive equation from a non-unique free energy functional. This relation will be the basis for the form of the constitutive relations given in subsection 3.5

For the static history $E^{\dagger}$, the quantity $\widetilde{S}\left(E^{t}, E(t)\right)$ in $(2.3)$ will be denoted by $\widetilde{S}_{e}(E(t))$ $=S_{e}(t)$, a function only of the current strain. Considering (2.7) for the static history, we see that

$$
\frac{d \widetilde{\phi}(E(t))}{d E(t)}=\frac{d \phi(t)}{d E(t)}=\widetilde{S}_{e}(E(t))=S_{e}(t) .
$$

Equation (2.12) for the static history yields that

$$
\widetilde{W}\left(E^{\dagger}, E(t)\right)=\int_{-\infty}^{t} \frac{d \phi(u)}{d E(u)} \dot{E}(u) d u=\phi(t),
$$

so that equality in (2.14) is achieved for the static history. It indeed holds only for the static history because this is true for any free energy $\psi(t)$, so that if $W(t)=\phi(t)$ for any other history, (2.14) would be contradicted.

Property P2 implies that

$$
\begin{aligned}
\psi(t) & =\phi(t)+\psi_{h}(t), \quad W(t)=\phi(t)+W_{h}(t), \quad \psi_{h}(t), W_{h}(t) \geq 0, \\
\psi_{h}(t) & =\widetilde{\psi}_{h}\left(E^{t}, E(t)\right) \geq 0, \quad W_{h}(t)=\widetilde{W}_{h}\left(E^{t}, E(t)\right) \geq 0,
\end{aligned}
$$

where $\psi_{h}(t)$ and $W_{h}(t)$ vanish only for the static history $E^{\dagger}$. Recalling (2.10), we see that to ensure the non-negativity of $\psi_{h}(t)$, the inequality

$$
\mathcal{D}(t) \leq W_{h}(t)
$$

must be true. Equality holds only for the static history or for a material with no memory. In these trivial cases, both sides of the inequality vanish.

REMARK 2.2. Equation (2.10) will prove to be a useful representation of any free energy, related in fact to that given on [2], page 117, where the fixed free energy in that equation is identified as the work function.

3. A generalized quadratic model for free energies. We now address the problem of finding explicit forms for free energies of materials which yield constitutive relations with memory functionals that are non-linear in a particular sense. The equilibrium term of these relations, which is the portion of the constitutive equation without memory effects, may be unrestrictedly non-linear. 
By means of a functional Taylor expansion to second order, we approximate $\widetilde{\psi}\left(E^{t}, E(t)\right)$ by the form ([2], for example)

$$
\begin{gathered}
\psi(t)=\phi(t)+\frac{1}{2} \int_{0}^{\infty} \int_{0}^{\infty} E_{r}^{t}(u) G_{12}(u, v ; E(t)) E_{r}^{t}(v) d u d v \\
=\phi(t)+\frac{1}{2} \int_{0}^{\infty} \int_{0}^{\infty} \dot{E}^{t}(u) \widetilde{G}(u, v ; E(t)) \dot{E}^{t}(v) d u d v \\
\dot{E}^{t}(u)=\frac{\partial}{\partial t} E^{t}(u)=-\frac{\partial}{\partial u} E^{t}(u)=-\frac{\partial}{\partial u} E_{r}^{t}(u),
\end{gathered}
$$

where

$$
\begin{aligned}
G_{12}(u, v ; E(t)) & =\frac{\partial^{2}}{\partial u \partial v} G(u, v ; E(t)), \\
\widetilde{G}(u, v ; E(t)) & =G(u, v ; E(t))-G_{\infty}(E(t)),
\end{aligned}
$$

and

$$
\lim _{u \rightarrow \infty} G(u, v ; E(t))=G_{\infty}(E(t)), \quad \lim _{u \rightarrow \infty} \frac{\partial}{\partial v} G(u, v ; E(t))=0, \quad u \in \mathbb{R}^{+},
$$

with similar limits at large $v$ holding for fixed $u$. Relation $3.3{ }_{1}$ is a definition of the quantity $G_{\infty}(E(t))$ used in $(3.2)_{2}$. Note that it is independent of $v$, which is a restriction on $G(u, v ; E(t))$. The quantity $G_{\infty}(E(t))$ is unique to the material and therefore not dependent on the choice of kernel $G(u, v ; E(t))$. This is clear from 3.10 below. Also,

$$
\lim _{u \rightarrow \infty} \frac{\partial}{\partial u} G(u, v ; E(t))=0, \quad v \in \mathbb{R}^{+}, \quad \lim _{v \rightarrow \infty} \frac{\partial}{\partial v} G(u, v ; E(t))=0, \quad u \in \mathbb{R}^{+} .
$$

Note that $(3.1)_{1}$ follows from $(3.1)_{2}$ by virtue of $(3.1)_{5}$.

The choice of $\phi(t)$, defined by (2.5) and (2.6), which provides a linear equilibrium term in the constitutive relations, has the form

$$
\phi(t)=\frac{1}{2} G_{\infty}(E(t)) E^{2}(t) .
$$

REMARK 3.1. The quantity $\phi(t)$ is always taken to be a non-negative function of $E(t)$, zero only if $E(t)=0$, while (2.8) gives that the integral terms in (3.1) are non-negative. From (3.1) 1 it is clear that the integral terms vanish for the static history. They are positive for all other histories. The kernels $\widetilde{G}$ and $G_{12}$ must be such that this property holds. Linear functional terms are omitted from the expression because they may take any sign.

Numerical subscripts will henceforth indicate differentiation with respect to the first or second argument, as in 3.2 .

REMARK 3.2. If the effect on the kernels of the variation of $E(t)$ can be neglected, we obtain the standard quadratic form for free energies, yielding linear memory terms in the constitutive relations [2]. For example, if $E(t)$ varies only to a small extent from a constant strain $E_{c}$, the kernel can be well approximated by $G\left(u, v, E_{c}\right)$, which yields a linear memory constitutive relation. A natural choice of $E_{c}$ is zero.

We refer to a theory based on such a kernel as a linear memory theory. The associated free energy will be referred to as a linear memory free energy, and so on for other quantities in the theory. 
This paper deals with materials where such dependence on $E(t)$ cannot be neglected.

Let us consider the kernel $G\left(u, v, E_{1}\right)$ for any arbitrary time-independent choice of the strain $E_{1}$ (which may be different from $E_{c}$ ) as that for a particular, known, linear memory free energy. We can in general write

$$
G(u, v ; E(t))=G\left(u, v, E_{1}\right)+G^{(E)}(u, v ; E(t)), \quad G^{(E)}\left(u, v ; E_{1}\right)=0 .
$$

The quantity $G\left(u, v, E_{1}\right)$ is assumed to be non-zero, since it is important that a term independent of $E(t)$ is present and the limit of a linear memory constitutive equation exists.

The simplest possibility for $G(u, v, E(t))$ is where it is a polynomial in $E(t)-E_{c}$, including a term independent of $E(t)$. The difference $E(t)-E_{c}$ is not now regarded as negligible. If the highest power of the polynomial is $N$, we refer to the model as of order $N$.

An example is the model of order two, given by

$$
G(u, v ; E(t))=G^{(0)}(u, v)+\left(E(t)-E_{c}\right)^{2} G^{(2)}(u, v) .
$$

The term proportional to $E(t)-E_{c}$ is omitted because there are positivity requirements on the kernel, at least for the quantity introduced in (3.9) below, and this linear term can take any sign. The quantities $G^{(i)}(u, v), i=1,2$, must be such that $G(u, v ; E(t))$ has the property imposed by (2.8) and referred to in Remark 3.1 for any choice of $E(t)$. It follows that both must be suitable kernels for linear memory free energies, in the sense of the non-negativity requirements deriving from (2.8).

Equation (3.1) can be written in the form (2], page 129)

$$
\begin{aligned}
\psi(t)=\sigma(t) & +\frac{1}{2} \int_{0}^{\infty} \int_{0}^{\infty} E^{t}(u) G_{12}(u, v ; E(t)) E^{t}(v) d u d v \\
\sigma(t)=\phi(t) & -\frac{1}{2}\left[G_{\infty}(E(t))-G_{0}(E(t))\right] E^{2}(t) \\
& +E(t) \int_{0}^{\infty} G^{\prime}(u ; E(t)) E^{t}(u) d u
\end{aligned}
$$

where

$$
G(u ; E(t))=G(0, u ; E(t))=G(u, 0 ; E(t)), \quad u \in \mathbb{R}^{+} .
$$

The left-hand side of this relation will be referred to as the relaxation function. It is a unique, given property of the material, the same for any choice of free energy kernel, so that (3.9) is a constraint on the $E(t)$ dependence, as well as the $u, v$ dependence of $G(u, v ; E(t))$. The relaxation function is generally assumed to be a non-negative quantity.

The prime on $G(u ; E(t))$ in $(3.8)_{2}$ indicates differentiation with respect to its first argument. Furthermore,

$$
G_{0}(E(t))=G(0 ; E(t)), \quad G_{\infty}(E(t))=\lim _{u \rightarrow \infty} G(u, E(t)) .
$$

Note that $G_{\infty}(E(t))$ is the same as the quantity introduced in $(3.3)_{1}$. For $\phi(t)$ given by (3.5),

$$
\sigma(t)=\frac{1}{2} G_{0}(E(t)) E^{2}(t)+E(t) \int_{0}^{\infty} G^{\prime}(u ; E(t)) E^{t}(u) d u .
$$


Corresponding to (3.6) and taking account of (3.9), we have

$$
G(u ; E(t))=G\left(u ; E_{1}\right)+G^{(E)}(u, E(t)), \quad G^{(E)}\left(u ; E_{1}\right)=0 .
$$

The model of order two, given by (3.7), corresponds to the form of the relaxation function

$$
\begin{aligned}
G(u ; E(t)) & =G^{(0)}(u)+\left(E(t)-E_{c}\right)^{2} G^{(2)}(u), \\
G^{(i)}(u) & =G^{(i)}(u, 0), \quad i=0,2 .
\end{aligned}
$$

The quantities $G^{(i)}(u)$ must be such that $G(u ; E(t))$ has the required properties for a relaxation function, in particular (4.5) below, for any value of $E(t)$. This requires that both these quantities are valid relaxation functions for a linear memory material, obeying (4.5).

REMARK 3.3. For even powered polynomial models, and indeed any model where $G(u, v ; E(t))$ diverges positively for large values of $|E(t)|$, a limit most be imposed on the size of $|E(t)|$, which we denote by $E_{l}>0$. For example, this could be slightly below a critical strain at which failure or a phase transition occurs. This limit will be directly invoked when discussing the minimum free energy in section 8 .

3.1. Forms of the work function. We now present various expressions for the total work done on the material, given by (2.12). Recalling Remark 2.1, we seek quadratic forms for $W(t)$ similar to (3.1) or (3.8) and for which the kernel $K$ of the rate of dissipation, given by (3.25) below, vanishes. Also, the linear memory result (2], page 153) must readily emerge. The only choices obeying these requirements are given by

$$
\begin{aligned}
W(t) & =\phi(t)+\frac{1}{2} \int_{0}^{\infty} \int_{0}^{\infty} G_{12}(|u-v| ; E(t)) E_{r}^{t}(u) E_{r}^{t}(v) d u d v \\
& =\sigma(t)+\frac{1}{2} \int_{0}^{\infty} \int_{0}^{\infty} G_{12}(|u-v| ; E(t)) E^{t}(u) E^{t}(v) d u d v \\
& =\phi(t)+\frac{1}{2} \int_{0}^{\infty} \int_{0}^{\infty} G(|u-v| ; E(t)) \dot{E}^{t}(u) \dot{E}^{t}(v) d u d v
\end{aligned}
$$

where the kernel is the relaxation function introduced in (3.9).

We will need the quantity $W(t+s)$, given by

$$
\begin{aligned}
W(t+s)=\sigma(t & +s)+\frac{1}{2} \int_{-s}^{\infty} \int_{-s}^{\infty} G_{12}(|u-v| ; E(t+s)) E^{t}(u) E^{t}(v) d u d v \\
=\sigma(t & +s)+\int_{0}^{\infty} \int_{0}^{s} G_{12}(u+y ; E(t+s)) E^{t}(u) E(t+y) d u d y \\
& +\frac{1}{2} \int_{0}^{s} \int_{0}^{s} G_{12}(w+y ; E(t+s)) E(t+w) E(t+y) d w d y \\
& +\frac{1}{2} \int_{0}^{\infty} \int_{0}^{\infty} G_{12}(|u-v| ; E(t+s)) E^{t}(u) E^{t}(v) d u d v .
\end{aligned}
$$


This follows by observing that $E^{t+s}(u)=E^{t}(u-s)$ and carrying out changes in the integration variables. Also, from (3.8),

$$
\begin{aligned}
\sigma(t+s)=\phi( & +s)-\frac{1}{2}\left[G_{\infty}(E(t+s))-G_{0}(E(t+s))\right] E^{2}(t+s) \\
& +E(t+s) \int_{0}^{\infty} G^{\prime}(u+s ; E(t+s)) E^{t}(u) d u \\
& +E(t+s) \int_{0}^{s} G^{\prime}(s-y ; E(t+s)) E(t+y) d y .
\end{aligned}
$$

Recalling the notation introduced in (2.12), we also define the functionals

$$
\begin{aligned}
\widetilde{W}\left(E^{t}, E_{0}\right) & =\widetilde{\phi}\left(E_{0}\right)+\frac{1}{2} \int_{0}^{\infty} \int_{0}^{\infty} G_{12}\left(|u-v| ; E_{0}\right) E_{0}^{t}(u) E_{0}^{t}(v) d u d v, \\
E_{0}^{t}(u) & =E^{t}(u)-E_{0} \\
W\left(t ; E_{0}\right) & =\phi(t)+\frac{1}{2} \int_{0}^{\infty} \int_{0}^{\infty} G_{12}\left(|u-v| ; E_{0}\right) E_{r}^{t}(u) E_{r}^{t}(v) d u d v
\end{aligned}
$$

where $E_{0}$ is a given strain, independent of $t$.

3.2. Dissipation. Using (3.1) $2,(3.14)_{3}$ and (2.10), we see that

$$
G(u, v ; E(t))+\Delta(u, v)=G(|u-v| ; E(t)),
$$

where $\Delta(u, v)$ is the kernel of the total dissipation. Relations (3.9), (3.18) and (3.3) 1 give that

$$
\begin{aligned}
\Delta(u, 0) & =\Delta(0, u)=0, \quad u \in \mathbb{R}^{+}, \\
\lim _{u \rightarrow \infty} \Delta(u, v) & =\lim _{u \rightarrow \infty} \Delta(v, u)=0, \quad v \in \mathbb{R}^{+} .
\end{aligned}
$$

The total dissipation has the form

$$
\begin{aligned}
\mathcal{D}(t) & =\frac{1}{2} \int_{0}^{\infty} \int_{0}^{\infty} \Delta(u, v) \dot{E}^{t}(u) \dot{E}^{t}(v) d u d v \\
& =\frac{1}{2} \int_{0}^{\infty} \int_{0}^{\infty} \Delta_{12}(u, v) E_{r}^{t}(u) E_{r}^{t}(v) d u d v
\end{aligned}
$$

where the second form requires $(3.1)_{5}$. Using (3.19), we find that $(3.20)_{2}$ becomes

$$
\mathcal{D}(t)=\frac{1}{2} \int_{0}^{\infty} \int_{0}^{\infty} \Delta_{12}(u, v) E^{t}(u) E^{t}(v) d u d v .
$$

Relations (2.18) and (2.19) are clearly true for this form.

Since $\Delta(u, v)$ in (3.18) is independent of $E(t)$, we can put

$$
\Delta(u, v)=G\left(|u-v| ; E_{1}\right)-G\left(u, v ; E_{1}\right),
$$

for any choice of $E_{1}$, and in particular for the parameter introduced in (3.6). Using this in (3.18), we obtain

$$
G(u, v ; E(t))=G\left(u, v ; E_{1}\right)+G(|u-v| ; E(t))-G\left(|u-v| ; E_{1}\right)
$$

or, recalling (3.6) and (3.12),

$$
G^{(E)}(u, v ; E(t))=G^{(E)}(|u-v| ; E(t))=G(|u-v| ; E(t))-G\left(|u-v| ; E_{1}\right) .
$$


These are significant restrictions on $G(u, v ; E(t))$. They ensure the uniqueness of the constitutive relations. Note that $\Delta(u, v)$ depends in general on the parameter $E_{1}$.

Using (2.11) $3,3.201,3$ (3.19) and (3.22), we obtain

$$
\begin{aligned}
D(t) & =D\left(t ; E_{1}\right)=-\frac{1}{2} \int_{0}^{\infty} \int_{0}^{\infty} \dot{E}^{t}(u) K(u, v) \dot{E}^{t}(v) d u d v \\
& =-\frac{1}{2} \int_{0}^{\infty} \int_{0}^{\infty} E_{r}^{t}(u) K_{12}(u, v) E_{r}^{t}(v) d u d v, \\
K(u, v) & =G_{1}\left(u, v ; E_{1}\right)+G_{2}\left(u, v ; E_{1}\right) .
\end{aligned}
$$

Note that the kernel $K(u, v)$ is independent of $E(t)$. However, we see from (3.25) 2 that $D\left(t ; E_{1}\right)$ may depend on $E(t)$.

3.3. General form of the free energy. By virtue of (3.23), we find that the general form of the free energy functional is

$$
\begin{aligned}
\psi(t) & =\psi_{l}\left(t ; E_{1}\right)+W(t ; E(t))-W\left(t ; E_{1}\right), \\
\psi_{l}\left(t ; E_{1}\right) & =\phi(t)+\frac{1}{2} \int_{0}^{\infty} \int_{0}^{\infty} G_{12}\left(u, v ; E_{1}\right) E_{r}^{t}(u) E_{r}^{t}(v) d u d v .
\end{aligned}
$$

The quantity $W\left(t ; E_{1}\right)$ is defined by (3.17) 3 . The free energy $\psi(t)$ will in general depend on the choice of $E_{1}$, so that we denote it by $\psi\left(t ; E_{1}\right)$.

REMARK 3.4. It is assumed that the quantity $\psi_{l}\left(t ; E_{1}\right)$ is a valid linear memory free energy with kernel $G_{12}\left(u, v ; E_{1}\right)$, which is independent of $E(t)$. The associated total dissipation is

$$
\mathcal{D}_{l}\left(t ; E_{1}\right)=W\left(t ; E_{1}\right)-\psi_{l}\left(t ; E_{1}\right) \geq 0,
$$

while the rate of dissipation $D_{l}\left(t ; E_{1}\right)$ is given by (3.25) and must be non-negative, by virtue of $\mathrm{P} 3$ for $\psi_{l}\left(t ; E_{1}\right)$.

The total dissipation relating to $\psi\left(t ; E_{1}\right)$ can be seen from (2.10) and (3.26) to be equal to $D_{l}\left(t ; E_{1}\right)$, so that

$$
\mathcal{D}(t)=\mathcal{D}\left(t ; E_{1}\right)=W\left(t ; E_{1}\right)-\psi_{l}\left(t ; E_{1}\right) \geq 0,
$$

which is independent of $E(t)$. The time derivative yields $D\left(t ; E_{1}\right)$, as given by (3.25).

3.4. Demonstration that $\psi\left(t ; E_{1}\right)$ is a free energy. We now show that $\psi\left(t ; E_{1}\right)$, given by (3.26), obeys the properties P1 - P3 listed in subsection 2.1 Property P1 follows from (2.17). The time derivative of (3.26) gives (2.9) or

$$
\begin{aligned}
\dot{\psi}\left(t ; E_{1}\right)+D\left(t ; E_{1}\right) & =S(t) \dot{E}(t), \\
D\left(t ; E_{1}\right) & =D_{l}\left(t ; E_{1}\right)=\dot{\mathcal{D}}_{l}\left(t ; E_{1}\right) \geq 0,
\end{aligned}
$$

as noted after (3.27). It follows that P3 is true also for $\psi\left(t ; E_{1}\right)$.

Property P2, which is clearly true for minimum and related free energies in the linear memory case, is not manifest for (3.26). However, it can be shown to be valid, as a consequence of the second law [3], expressed in this context by (3.29) 2 . Intuitively, this can be seen by considering a history which, after $t=0$, is given by $E(t)=E(0)$. At large times, this is approximately a static history so that $\psi\left(t ; E_{1}\right) \approx \phi(t)$. The right-hand side of $(3.29) 1$ vanishes for $t>0$, so that the time derivative of $\psi\left(t ; E_{1}\right)$ is negative. Thus, $\psi\left(t ; E_{1}\right) \geq \phi(t)$. 
3.5. Constitutive relations. Using (2.7), one obtains the following forms of the stress function:

$$
\begin{aligned}
S(t)= & \widetilde{S}_{e}(E(t))+\int_{0}^{\infty} G^{\prime}(u ; E(t)) E_{r}^{t}(u) d u \\
& \quad+\frac{1}{2} \int_{0}^{\infty} \int_{0}^{\infty} E_{r}^{t}(u) G_{12 E}(|u-v| ; E(t)) E_{r}^{t}(v) d u d v \\
= & \widetilde{S}_{e}(E(t))+\int_{0}^{\infty} \widetilde{G}(u ; E(t)) \dot{E}^{t}(u) d u \\
& \quad+\frac{1}{2} \int_{0}^{\infty} \int_{0}^{\infty} \dot{E}^{t}(u) \widetilde{G}_{E}(|u-v| ; E(t)) \dot{E}^{t}(v) d u d v \\
= & \widetilde{S}_{0}\left(E^{t}, E(t)\right)+\frac{1}{2} \int_{0}^{\infty} \int_{0}^{\infty} E^{t}(u) G_{12 E}(|u-v| ; E(t)) E^{t}(v) d u d v
\end{aligned}
$$

where $\widetilde{S}_{e}(E(t))$ is defined by (2.21). Also,

$$
\begin{aligned}
\widetilde{G}(u ; E(t)) & =G(u ; E(t))-G_{\infty}(E(t)) \\
\widetilde{S}_{0}\left(E^{t}, E(t)\right) & =\frac{\partial \sigma(t)}{\partial E(t)}=\widetilde{S}_{e}(E(t))-\left[G_{\infty}(E(t))-G_{0}(E(t))\right] E(t) \\
& +\int_{0}^{\infty} G^{\prime}(u ; E(t)) E^{t}(u) d u+E(t) \int_{0}^{\infty} G_{E}^{\prime}(u ; E(t)) E^{t}(u) d u
\end{aligned}
$$

where the second relation follows from (3.8). Furthermore,

$$
\begin{gathered}
G_{E}(|u-v| ; E(t))=\frac{\partial}{\partial E(t)} G(|u-v| ; E(t)), \\
G_{12 E}(|u-v| ; E(t))=\frac{\partial}{\partial E(t)} G_{12}(|u-v| ; E(t)) .
\end{gathered}
$$

In general, the subscript $E$, attached to any quantity, as in (3.32), will indicate partial differentiation with respect to $E(t)$ (or $E(t+s)$ in many cases), the argument being omitted for brevity.

The forms given by (3.30) differ from what is known as finite linear viscoelasticity [4, 10] by the extra terms involving the derivative with respect to $E(t)$ of the kernels in the quadratic memory terms. They are also clearly derivable from a free energy functional.

REMARK 3.5. For polynomial models introduced in Remark 3.2 with the order two example given by (3.13), consider the extra, quadratic terms in (3.30). Putting $E_{c}=0$, we see that the leading terms in the kernels are proportional to $E(t)$ so that these extra contributions are cubic in the strain, and therefore of odd signature. This is in accord with physical intuition. If $E_{c}$ is not zero, the signature of the term proportional to this quantity is even.

4. Frequency domain quantities. We now consider the frequency domain representations of $\widetilde{G}(s ; E(t))$, defined by $(3.31)$, and $G^{\prime}(s ; E(t))$, which was introduced in (3.8). Similar representations of the strain history are also presented. 
4.1. Frequency domain representations of the material functions. The Fourier transforms of the quantities $\widetilde{G}(s ; E(t))$ and $G^{\prime}(s ; E(t))$ have the forms

$$
\begin{aligned}
& \widetilde{G}_{+}(\omega ; E(t))=\int_{0}^{\infty} \widetilde{G}(s ; E(t)) e^{-i \omega s} d s=\overline{\widetilde{G}_{+}}(-\omega ; E(t)), \\
& G_{+}^{\prime}(\omega ; E(t))=\int_{0}^{\infty} G^{\prime}(s ; E(t)) e^{-i \omega s} d s=\overline{G_{+}^{\prime}}(-\omega ; E(t)) .
\end{aligned}
$$

These are analytic in the lower half of the $\omega$ plane 2. We also assume that they are analytic on an open region including the real axis, and analytic at infinity. Let

$$
\begin{aligned}
G_{+}^{\prime}(\omega ; E(t)) & =G_{c}^{\prime}(\omega ; E(t))-i G_{s}^{\prime}(\omega ; E(t)), \\
G_{c}^{\prime}(\omega ; E(t)) & =G_{c}^{\prime}(-\omega ; E(t)), \quad G_{s}^{\prime}(\omega ; E(t))=-G_{s}^{\prime}(-\omega ; E(t)) .
\end{aligned}
$$

Similar relations apply to $\widetilde{G}_{+}(\omega ; E(t))$. We assume that

$$
\widetilde{G}, G^{\prime} \in L^{1}\left(\mathbb{R}_{+}\right) \cap L^{2}\left(\mathbb{R}^{+}\right),
$$

giving

$$
\widetilde{G}_{+}, G_{+}^{\prime} \in L^{2}(\mathbb{R}) .
$$

Various properties of $\widetilde{G}_{+}(\omega ; E(t))$ and $G_{+}^{\prime}(\omega ; E(t))$ can be derived using the same steps as for the linear case, where the $E(t)$ dependence is neglected (for example, 2], page $142)$.

The quantity $G_{s}^{\prime}(\omega ; E(t))$ has singularities in $\omega$ on both $\Omega^{(+)}$and $\Omega^{(-)}$and is analytic on the real axis. It vanishes linearly at the origin. By virtue of the second law, it has the property that

$$
G_{s}^{\prime}(\omega ; E(t)) \leq 0, \quad \forall \omega \geq 0 .
$$

An important quantity for our considerations is defined by

$$
H(\omega ; E(t))=-\omega G_{s}^{\prime}(\omega ; E(t)) \geq 0, \omega \in \mathbb{R} .
$$

It is a non-negative, even function of the frequency, vanishing quadratically at the origin. It can be shown that

$$
G^{\prime}(0 ; E(t))=-H(\infty ; E(t)) \equiv-H_{\infty}(E(t)), \quad H_{\infty}(E(t)) \geq 0 .
$$

We shall replace the parameter $E(t)$ in the various kernels by an arbitrary strain $E_{a}$, which is understood to include $E_{a}=E(t)$ or indeed $E(t+s), s \geq 0$.

For the model of order two, introduced in (3.7) and (3.13), we have, for $E_{c}=0$,

$$
H\left(\omega ; E_{a}\right)=H^{(0)}(\omega)+E_{a}^{2} H^{(2)}(\omega), \quad H^{(i)}(\omega) \geq 0, \quad i=0,2 .
$$

The non-negativity of these quantities follows from the observation after (3.13). It will be required to factorize the quantity $H\left(\omega ; E_{a}\right)$ in order to determine an expression for the minimum and other free energies. On the basis of a general result [2, 7, it can be shown that $H\left(\omega ; E_{a}\right)$ may be factorized as follows:

$$
H\left(\omega ; E_{a}\right)=H_{+}\left(\omega ; E_{a}\right) H_{-}\left(\omega ; E_{a}\right),
$$

where

$$
H_{ \pm}\left(\omega ; E_{a}\right)=H_{\mp}\left(-\omega ; E_{a}\right)=\overline{H_{\mp}}\left(\omega ; E_{a}\right) .
$$


The quantities $H_{ \pm}\left(\omega ; E_{a}\right)$ are analytic for $\omega$ in $\Omega^{(\mp)}$. By assumption, we extend this analyticity to open sets including the real axis, in both cases. Also, all the zeros of $H_{ \pm}\left(\omega ; E_{a}\right)$ are in $\Omega^{( \pm)}$as a function of $\omega$, respectively. These factors vanish linearly in $\omega$ at the origin. An explicit method for determining them is given in 2, 12]. They are unique up to a sign.

For the model of order two, as expressed by (4.8), the factors of $H\left(\omega ; E_{a}\right)$ are given by

$$
H_{ \pm}\left(\omega ; E_{a}\right)=H_{ \pm}^{(0)}(\omega)+E_{a} H_{ \pm}^{(2)}(\omega)
$$

and

$$
H\left(\omega ; E_{a}\right)=\left|H_{+}^{(0)}(\omega)\right|^{2}+E_{a}^{2}\left|H_{+}^{(2)}(\omega)\right|^{2},
$$

where, comparing with (4.8),

$$
H^{(i)}(\omega)=\left|H_{ \pm}^{(i)}(\omega)\right|^{2}, \quad i=0,2,
$$

if

$$
H_{+}^{(0)}(\omega) H_{-}^{(2)}(\omega)+H_{+}^{(2)}(\omega) H_{-}^{(0)}(\omega)=2 R e H_{+}^{(0)}(\omega) H_{-}^{(2)}(\omega)=0
$$

holds. Relation (4.14) states that $H_{+}^{(0)}(\omega) H_{-}^{(2)}(\omega)$ is imaginary. Thus, the quantities $H_{ \pm}^{(i)}(\omega), i=0,2$, are determined by factorizing $H^{(i)}(\omega)$ in such a manner that the $H_{ \pm}^{(i)}(\omega)$ have all their singularities in $\Omega^{ \pm}$respectively. There are no simple conditions on the zeros of $H_{ \pm}^{(i)}(\omega)$, except that they must be such that the zeros of $H_{ \pm}\left(\omega ; E_{a}\right)$ are in $\Omega^{ \pm}$. The quantities $H_{ \pm}^{(i)}(\omega)$ will be arbitrary up to certain phase factors which can be chosen so that $H_{+}^{(0)}(\omega) H_{-}^{(2)}(\omega)$ is imaginary. This property is assured by the fact that it is always possible to factorize $H\left(\omega ; E_{a}\right)$, as described by (4.9) and (4.10).

4.2. Frequency domain representation of the history. These formulae are unchanged from the linear theory [2]. We summarize here those that are needed later. Consider the Fourier transform of $E^{t} \in L^{1}\left(\mathbb{R}^{+}\right) \cap L^{2}\left(\mathbb{R}^{+}\right)$, given by

$$
E_{+}^{t}(\omega)=\int_{0}^{\infty} E^{t}(u) e^{-i \omega u} d u .
$$

The derivative of $E_{+}^{t}$ with respect to $t$ will be required. Assuming that $E^{t} \in C^{1}\left(\mathbb{R}^{+}\right)$ we find, with the aid of a partial integration, that

$$
\frac{d}{d t} E_{+}^{t}(\omega)=-i \omega E_{+}^{t}(\omega)+E(t)
$$

The Fourier transform of $E_{r}^{t}(u)=E^{t}(u)-E(t), u \in \mathbb{R}^{+}$, is given by

$$
E_{r+}^{t}(\omega)=E_{+}^{t}(\omega)-\frac{E(t)}{i \omega^{-}}
$$

which, on using the limit

$$
\lim _{\omega \rightarrow \infty} i \omega E_{+}^{t}(\omega)=E(t)
$$

can be seen to behave as $\omega^{-2}$ at large frequencies. The notation $\omega^{-}$(and $\omega^{+}$below) are defined in [2], page 551 . 
Application of the convolution theorem and Parseval's formula to (3.14) $)_{1,2}$ give ([2], page 154)

$$
\begin{aligned}
W(t) & =\phi(t)+\frac{1}{2 \pi} \int_{-\infty}^{\infty} \overline{E_{r+}^{t}}(\omega) H(\omega ; E(t)) E_{r+}^{t}(\omega) d \omega \\
& =\sigma(t)+\frac{1}{2 \pi} \int_{-\infty}^{\infty} \overline{E_{+}^{t}}(\omega) H(\omega ; E(t)) E_{+}^{t}(\omega) d \omega,
\end{aligned}
$$

in terms of the quantity defined by (4.6). Also, from (3.17),

$$
\begin{aligned}
\widetilde{W}\left(E^{t}, E_{0}\right) & =\widetilde{\phi}\left(E_{0}\right)+\frac{1}{2 \pi} \int_{0}^{\infty} \int_{0}^{\infty} H\left(\omega ; E_{0}\right) \overline{E_{0+}^{t}}(\omega) E_{0+}^{t}(\omega) d \omega, \\
E_{0+}^{t}(\omega) & =E_{+}^{t}(\omega)-\frac{E_{0}}{i \omega^{-}}, \\
W\left(t ; E_{0}\right) & =\phi(t)+\frac{1}{2 \pi} \int_{-\infty}^{\infty} \overline{E_{r+}^{t}}(\omega) H\left(\omega ; E_{0}\right) E_{r+}^{t}(\omega) d \omega \\
& =\sigma(t)+\frac{1}{2 \pi} \int_{-\infty}^{\infty} \overline{E_{+}^{t}}(\omega) H\left(\omega ; E_{0}\right) E_{+}^{t}(\omega) d \omega .
\end{aligned}
$$

Note that $E_{0}^{t}(0) \neq 0$ and $E_{0+}^{t}(\omega)$ behaves as $\omega^{-1}$ at large frequencies, in contrast to $E_{r+}^{t}(\omega)$, defined by (4.17).

5. Minimal states. In the classical approach to materials with memory, the state is identified with the history of the independent variables. Noll's characterization of state [18] is also of interest. He takes the material response as the basis for the definition of state: if an arbitrary process, acting on different given histories at time $t$, leads to the same response of the material after time $t$, then the given histories are equivalent and the state is represented as the class of all these equivalent histories. We shall refer to it as the minimal state 11 .

A minimal state is in effect an equivalence class under this definition. The idea has been applied to completely linear materials in for example [1,5, 9, 11, 16. The definition of a minimal state is now discussed in the present context. Equivalent states have the same current value $E(t)$. The two states $\left(E_{1}^{t}, E(t)\right),\left(E_{2}^{t}, E(t)\right)$ are equivalent or in the same minimal state if

$$
S_{1}(t+s)=S_{2}(t+s), \quad E_{1}(t+s)=E_{2}(t+s), \quad s \geq 0,
$$

where $S_{1}, S_{2}$ are the stresses corresponding to the states $\left(E_{1}^{t}, E(t)\right),\left(E_{2}^{t}, E(t)\right)$, respectively. If the minimal state is a singleton, then $E_{1}^{t}(s)=E_{2}^{t}(s), s \geq 0$. Otherwise, the histories may be different.

REMARK 5.1. We can regard the history $E_{1}^{t}$ as given and find the set of histories $E_{2}^{t}$ which are consistent with (5.1). This is the minimal state containing the history $E_{1}^{t}$.

REMARK 5.2. It will become clear below that the condition (5.1 $)_{1}$ takes a complicated form, difficult to meaningfully analyze. A method of avoiding this difficulty is to formulate simpler conditions which imply $(5.1)_{1}$, but which may not be equivalent to it. What this means, in effect, is that we would extract minimal states which are subsets, possibly proper subsets, of those that would emerge from a fully general analysis of (5.1). 
A functional of $\left(E^{t}, E(t)\right)$ which yields the same value for all members of the same minimal state will be referred to as a functional of the minimal state or a state functional or indeed a minimal state variable.

We have from $(3.30)_{3}$ and $(3.31)_{2}$ that

$$
\begin{aligned}
S(t+s)= & \widetilde{S}_{e}(E(t+s))-\left[G_{\infty}(E(t+s))-G_{0}(E(t+s))\right] E(t+s) \\
& +\int_{0}^{\infty} G^{\prime}(u ; E(t+s)) E^{t+s}(u) d u \\
& +E(t+s) \int_{0}^{\infty} G_{E}^{\prime}(u ; E(t+s)) E^{t+s}(u) d u \\
& +\frac{1}{2} \int_{0}^{\infty} \int_{0}^{\infty} G_{12 E}(|u-v| ; E(t+s)) E^{t+s}(u) E^{t+s}(v) d u d v \\
= & \widetilde{S}_{e}(E(t+s))-\left[G_{\infty}(E(t+s))-G_{0}(E(t+s))\right] E(t+s) \\
& +\int_{-s}^{\infty} G^{\prime}(u+s ; E(t+s)) E^{t}(u) d u \\
& +E(t+s) \int_{-s}^{\infty} G_{E}^{\prime}(u+s ; E(t+s)) E^{t}(u) d u \\
& +\frac{1}{2} \int_{-s}^{\infty} \int_{-s}^{\infty} G_{12 E}(|w-z| ; E(t+s)) E^{t}(w) E^{t}(z) d w d z
\end{aligned}
$$

giving

$$
\begin{aligned}
S_{1}(t+ & s)-S_{2}(t+s)=\int_{0}^{\infty} G^{\prime}(s+u ; E(t+s)) E_{d}^{t}(u) d u \\
& +E(t+s) \int_{0}^{\infty} G_{E}^{\prime}(s+u ; E(t+s)) E_{d}^{t}(u) d u \\
& +\int_{0}^{\infty} \int_{0}^{s} G_{12 E}(w+y ; E(t+s)) E_{d}^{t}(w) E(t+y) d w d y \\
& +\frac{1}{2} \int_{0}^{\infty} \int_{0}^{\infty} G_{12 E}(|w-z| ; E(t+s)) E_{1}^{t}(w) E_{1}^{t}(z) d w d z \\
& -\frac{1}{2} \int_{0}^{\infty} \int_{0}^{\infty} G_{12 E}(|w-z| ; E(t+s)) E_{2}^{t}(w) E_{2}^{t}(z) d w d z
\end{aligned}
$$

where

$$
E_{d}^{t}(u)=E_{1}^{t}(u)-E_{2}^{t}(u), \quad E(t+s)=E_{1}(t+s)=E_{2}(t+s) .
$$

The double integrals where both integrals are over $(0,-s)$ cancel as a result of $(5.1)_{2}$, as do the single integrals over the same interval. The double integrals where one integral is over $(0,-s)$ have been combined and the integration transformed to $(0, s)$. Equation 
(5.3) can be written in the form

$$
\begin{gathered}
S_{1}(t+s)-S_{2}(t+s)=\int_{0}^{\infty} L(s, u) E_{d}^{t}(u) d u \\
+\int_{0}^{\infty} N(s,|w-z|)\left[E_{1}^{t}(w) E_{1}^{t}(z)-E_{2}^{t}(w) E_{1}^{t}(z)\right] d w d z \\
L(s, u)=\frac{\partial}{\partial E(t+s)}\left[E(t+s) G^{\prime}(u+s, E(t+s))\right] \\
\quad+\int_{0}^{s} G_{E}^{\prime \prime}(u+y ; E(t+s)) E_{d}^{t}(w) E(t+y) d y \\
N(s,|w-z|)=\frac{1}{2} G_{12 E}(|w-z| ; E(t+s)) .
\end{gathered}
$$

The general form of (5.1) 1 can therefore be expressed in the form

$$
\begin{aligned}
& \int_{0}^{\infty} L(s, u) E_{2}^{t}(u) d u+\int_{0}^{\infty} N(s,|w-z|) E_{2}^{t}(w) E_{2}^{t}(z) d w d z \\
& =\int_{0}^{\infty} L(s, u) E_{1}^{t}(u) d u+\int_{0}^{\infty} N(s,|w-z|) E_{1}^{t}(w) E_{1}^{t}(z) d w d z
\end{aligned}
$$

for $s \geq 0$, where the right-hand side is known. This is a non-linear integral equation which determines the set of histories $E_{2}^{t}$ forming the minimal state containing the given history $E_{1}^{t}$. However, the solution of (5.6) is clearly a difficult task, and so the procedure outlined in Remark 5.2 will be followed.

Observe that if $G^{\prime}$ does not depend on $E(t+s)$, the right-hand side of (5.3) reduces to the first term, which, with $E(t+s)$ dependence included, we write as

$$
I_{d}^{t}(s, E(t+s))=I_{1}^{t}(s, E(t+s))-I_{2}^{t}(s, E(t+s)),
$$

where $I_{i}^{t}(s, E(t+s)), i=1,2$, denote the quantity

$$
I^{t}(s ; E(t+s))=\int_{0}^{\infty} G^{\prime}(s+u ; E(t+s)) E^{t}(u) d u, \quad s \geq 0,
$$

for histories $E_{1}^{t}$ and $E_{2}^{t}$ respectively. The functional $I^{t}(s ; E(t+s))$ is a special case of the more general quantity

$$
I^{t}(y ; E(t+s))=\int_{0}^{\infty} G^{\prime}(y+u ; E(t+s)) E^{t}(u) d u, \quad y, s \geq 0 .
$$

Also, generalizing (5.7), we put

$$
I_{d}^{t}(y ; E(t+s))=\int_{0}^{\infty} G^{\prime}(y+u ; E(t+s)) E_{d}^{t}(u) d u, \quad y, s \geq 0 .
$$

The first double integral in (5.3), with $E_{d}^{t}(w)$ replaced by $E^{t}(w)$, can be written as

$$
\begin{aligned}
\int_{0}^{\infty} \int_{0}^{s} G_{E}^{\prime \prime} & (w+y ; E(t+s)) E^{t}(w) E(t+y) d w d y \\
& =\int_{0}^{s} I_{(1) E}^{t}(y ; E(t+s)) E(t+y) d y, \\
I_{(1)}^{t}(y ; E(t+s)) & =\frac{\partial}{\partial y} I^{t}(y ; E(t+s)) .
\end{aligned}
$$


Let us define

$$
\begin{aligned}
J^{t}(s ; E(t+s))= & \frac{\partial}{\partial E(t+s)}\left[E(t+s) I^{t}(s, E(t+s))\right] \\
& +\int_{0}^{s} I_{(1) E}^{t}(y ; E(t+s)) E(t+y) d y \\
M^{t}(E(t+s))= & \frac{1}{2} \int_{0}^{\infty} \int_{0}^{\infty} G_{12}(|w-z| ; E(t+s)) E^{t}(w) E^{t}(z) d w d z \\
M_{E}^{t}(E(t+s))= & \frac{\partial}{\partial E(t+s)} M^{t}(E(t+s)) \\
= & \frac{1}{2} \int_{0}^{\infty} \int_{0}^{\infty} G_{12 E}(|w-z| ; E(t+s)) E^{t}(w) E^{t}(z) d w d z \\
T^{t}(s ; E(t+s))= & J^{t}(s ; E(t+s))+M_{E}^{t}(E(t+s)) .
\end{aligned}
$$

If two states $\left(E_{1}(t), T_{1}^{t}\left(s, E_{1}(t+s)\right)\right)$ and $\left(E_{2}(t), T_{2}^{t}\left(s, E_{2}(t+s)\right)\right)$ are such that

$$
\begin{aligned}
E_{1}(t+s) & =E_{2}(t+s), \\
T_{1}^{t}(s ; E(t+s)) & =T_{2}^{t}(s ; E(t+s)), \quad s \geq 0,
\end{aligned}
$$

where the subscripts on $T_{1}$ and $T_{2}$ indicate that these are functionals of the histories $E_{1}^{t}$ and $E_{2}^{t}$, respectively, then both are in the same minimal state. The fundamental minimal state variable is $T^{t}(s ; E(t+s))$. Relation (5.13) $)_{2}$ is equivalent to (5.6) or (5.1) 1 .

Referring to Remark 5.2, we see that a natural way of satisfying $(\underline{5.13})_{2}$ is to impose the possibly stronger conditions

$$
\begin{aligned}
& J_{1}^{t}(s, E(t+s))=J_{2}^{t}(s, E(t+s)), \\
& M_{1 E}^{t}(E(t+s))=M_{2 E}^{t}(E(t+s)), \quad s \geq 0 .
\end{aligned}
$$

Following the convention of (5.7), we put

$$
\begin{gathered}
J_{d}^{t}(s, E(t+s))=J_{1}^{t}(s, E(t+s))-J_{2}^{t}(s, E(t+s)), \\
M_{d}^{t}(E(t+s))=M_{1}^{t}(E(t+s))-M_{2}^{t}(E(t+s)) .
\end{gathered}
$$

Relation (5.14) 1 is the condition $J_{d}^{t}(s, E(t+s))=0$, which becomes an equation for $I_{d}^{t}(s, E(t+s))$ of the form

$$
\frac{\partial}{\partial E(t+s)}\left[E(t+s) I_{d}^{t}(s, E(t+s))\right]+\int_{0}^{s} I_{d(1) E}^{t}(y ; E(t+s)) E(t+y) d y=0 .
$$

By a further application of the procedure described in Remark 5.2 , we replace this equation by a condition on $I_{d}^{t}(y, E(t+s))$ and replace (5.14) by

$$
I_{d}^{t}(y, E(t+s))=0, \quad M_{d E}(E(t+s))=0, \quad y, s \geq 0 .
$$

An alternative form of these constraints is

$$
I_{d}^{t}(y, E(t+s))=0, \quad S_{1}(t+s)=S_{2}(t+s), \quad y, s \geq 0
$$


given that $(5.13)_{2}$ is equivalent to (5.1) 1 . Noting that $(3.14)_{2}$ becomes $(4.19)_{2}$, we see, by the same argument, that 5.12$)_{2,3,4}$ can be put in the form

$$
\begin{aligned}
& M^{t}(E(t+s))=\frac{1}{2 \pi} \int_{-\infty}^{\infty} \overline{E_{+}^{t}}(\omega) H(\omega ; E(t+s)) E_{+}^{t}(\omega) d \omega, \\
& M_{E}^{t}(E(t+s))=\frac{1}{2 \pi} \int_{-\infty}^{\infty} \overline{E_{+}^{t}}(\omega) H_{E}(\omega ; E(t+s)) E_{+}^{t}(\omega) d \omega,
\end{aligned}
$$

and $(5.17)_{2}$ becomes

$$
\begin{aligned}
\int_{-\infty}^{\infty} \overline{E_{1+}^{t}}(\omega) H_{E}(\omega ; E(t+s)) E_{1+}^{t}(\omega) d \omega \\
\quad=\int_{-\infty}^{\infty} \overline{E_{2+}^{t}}(\omega) H_{E}(\omega ; E(t+s)) E_{2+}^{t}(\omega) d \omega, \quad s \geq 0 .
\end{aligned}
$$

REMARK 5.3. The continuation $E(t+s), s \geq 0$, is arbitrary, so that we can put $E(t+s)=E_{a}$ where $E_{a}$ is an arbitrary strain and (5.17) $)_{1}$ becomes

$$
\left.I_{1}^{t}\left(y, E_{a}\right)\right)-I_{2}^{t}\left(y, E_{a}\right)=\int_{0}^{\infty} G^{\prime}\left(u+y ; E_{a}\right) E_{d}^{t}(u) d u=0, \quad y \geq 0 .
$$

Also, (5.17) 2 or (5.20) must hold for $E(t+s)=E_{a}$.

Proposition 5.1. Consider two equivalent states $\left(E_{1}^{t}, E(t)\right)$ and $\left(E_{2}^{t}, E(t)\right)$. Let

$$
\begin{gathered}
\psi_{1}(t+s)=\widetilde{\psi}\left(E_{1}^{t+s}, E(t+s)\right), \quad \psi_{2}(t+s)=\widetilde{\psi}\left(E_{2}^{t+s}, E(t+s)\right), \\
\widetilde{F}\left(E_{1}^{t+s}, E_{2}^{t+s}, E(t+s)\right)=\psi_{1}(t+s)-\psi_{2}(t+s),
\end{gathered}
$$

where $\widetilde{\psi}\left(E^{t+s}, E(t+s)\right)$ is any free energy functional, including the work function $\widetilde{W}\left(E^{t+s}, E(t+s)\right)$. Then, the quantity $\widetilde{F}\left(E_{1}^{t+s}, E_{2}^{t+s}, E(t+s)\right)$ is independent of $E(t+s)$ or

$$
\frac{\partial}{\partial E(t+s)} \widetilde{F}\left(E_{1}^{t+s}, E_{2}^{t+s}, E(t+s)\right)=\widetilde{F}_{E}\left(E_{1}^{t+s}, E_{2}^{t+s}, E(t+s)\right)=0, \quad s \geq 0,
$$

which is equivalent to

$$
\widetilde{F}\left(E_{1}^{t+s}, E_{2}^{t+s}, E(t+s)\right)=\widetilde{F}\left(E_{1}^{t+s}, E_{2}^{t+s}, E_{a}\right),
$$

where $E_{a}$ is any strain, equal to or different from $E(t+s)$.

Proof. This follows immediately from (2.7) and (5.1) 1 .

Note that (5.23) holds for general non-linear theories.

For the model given by (3.13), (5.17) 2 yields the condition

$$
\begin{aligned}
& \int_{0}^{\infty} \int_{0}^{\infty} G_{12}^{(2)}(|u-v|) E_{1}^{t}(u) E_{1}^{t}(v) d u d v \\
& \quad=\int_{0}^{\infty} \int_{0}^{\infty} G_{12}^{(2)}(|u-v|) E_{2}^{t}(u) E_{2}^{t}(v) d u d v .
\end{aligned}
$$

The condition (5.17) 1 gives

$$
\int_{0}^{\infty} G^{(i) \prime}(u+s) E_{d}^{t}(u) d s d u=0, \quad s \geq 0, \quad i=0,2,
$$


where $E_{d}^{t}(u)$ is defined by (5.4) $)_{1}$. These are two conditions analogous to that for a linear theory. The extra conditions represented by (5.25) and one of those in (5.26) will tend to reduce the size of the minimal state.

Let $\left(E_{1}^{t}, E(t)\right),\left(E_{2}^{t}, E(t)\right)$ be any equivalent states. Then, a free energy is a functional of the minimal state if

$$
\widetilde{\psi}\left(E_{1}^{t}, E(t)\right)=\widetilde{\psi}\left(E_{2}^{t}, E(t)\right) .
$$

It is not necessary that a free energy have this property, though it holds for most of the free energies of interest in the present work.

Proposition 5.2. If $\psi_{l}\left(t ; E_{1}\right)$ in (3.26) is a linear memory free energy that is a functional of the minimal state, as defined by (5.27), where the equivalence condition has the linear memory theory form (5.17) 1 for $E(t+s)=E_{1}$, then $\psi(t)=\psi\left(t ; E_{1}\right)$ will also have this property for the non-linear equivalence conditions (5.17).

Proof. We seek to show that

$$
W_{1}(t ; E(t))-W_{1}\left(t ; E_{a}\right)=W_{2}(t ; E(t))-W_{2}\left(t ; E_{a}\right),
$$

using notation from (2.12) and (3.17) 3 . Also, as before, the subscripts on $W_{1}$ and $W_{2}$ imply that they are functionals of $E_{1}^{t}$ and $E_{2}^{t}$, respectively. We temporarily use $E_{a}$ here instead of $E_{1}$ to avoid confusion with the notation $E_{1}^{t}$. By virtue of Proposition [5.1 applied to work functions, we have, putting $s=0$ in (5.24),

$$
\begin{aligned}
\widetilde{W}\left(E_{1}^{t}, E(t)\right)-\widetilde{W}\left(E_{2}^{t}, E(t)\right) & =W_{1}(t ; E(t))-W_{2}(t ; E(t)) \\
& =\widetilde{W}\left(E_{1}^{t}, E_{a}\right)-\widetilde{W}\left(E_{2}^{t}, E_{a}\right),
\end{aligned}
$$

where the first relation is simply a change in notation (see (2.12) ) and the quantities on the right of $(\underline{5.29})_{2}$ are defined by $(3.17)_{1,2}$. Also

$$
\begin{aligned}
\widetilde{W}\left(E_{1}^{t}, E_{a}\right)=W_{1}( & \left.t, E_{a}\right)-\widetilde{\phi}(E(t))+\widetilde{\phi}\left(E_{a}\right) \\
& -\left[E(t)-E_{a}\right] \int_{0}^{\infty} G^{\prime}\left(v ; E_{a}\right) E_{1 r}^{t}(v) d v \\
+ & \frac{1}{2}\left[E(t)-E_{a}\right]^{2}\left[G_{0}\left(E_{a}\right)-G_{\infty}\left(E_{a}\right)\right],
\end{aligned}
$$

so that

$$
\begin{aligned}
\widetilde{W}\left(E_{1}^{t}, E_{a}\right)-\widetilde{W}\left(E_{2}^{t}, E_{a}\right)= & W_{1}\left(t, E_{a}\right)-W_{2}\left(t, E_{a}\right) \\
& -\left[E(t)-E_{a}\right] \int_{0}^{\infty} G^{\prime}\left(v ; E_{a}\right) E_{d}^{t}(v) d v .
\end{aligned}
$$

The final term vanishes by virtue of $(5.17)_{1}$ or (5.18) 1 for $y=0$, so that (5.31) and (5.29) yield (5.28). This completes the demonstration that $\psi\left(t ; E_{a}\right)=\psi\left(t ; E_{1}\right)$ is a functional of the minimum state.

Note that (5.29) is equivalent to (5.23) for free energies equal to the work function, with $s=0$. This is $(5.18)_{2}$ for $s=0$. Thus, invoking Proposition 5.1 is equivalent to using (5.18) 2 so that both relations of (5.18) or (5.17) are used in the above proof. 
6. Minimal states for discrete spectrum materials. More specific observations can be made on the issue of minimal states within a particular material model. Let the relaxation function $G\left(t ; E_{a}\right)$ have the form

$$
G\left(s ; E_{a}\right)=G_{\infty}\left(E_{a}\right)+\sum_{i=1}^{n} G_{i}\left(E_{a}\right) e^{-\alpha_{i} s}, \quad G_{\infty}\left(E_{a}\right) \geq 0,
$$

where $n$ is a positive integer, the inverse decay times $\alpha_{i} \in \mathbb{R}^{++}, i=1,2, \ldots, n$, while the coefficients $G_{i}$ are also generally assumed to be positive. Materials with such relaxation functions are often described as discrete spectrum materials (2], page 264) and provide simple examples for illustrating some concepts and results presented in this work. Note that we allow the coefficients $G_{i}$ to depend on $E_{a}$, but not the quantities $\alpha_{i}$, which achieves some simplification. We arrange that $\alpha_{1}<\alpha_{2}<\alpha_{3} \ldots$ Taking the derivative with respect to $s$, we obtain

$$
G^{\prime}\left(s ; E_{a}\right)=\sum_{i=1}^{n} g_{i}\left(E_{a}\right) e^{-\alpha_{i} s}, \quad g_{i}\left(E_{a}\right)=-\alpha_{i} G_{i}\left(E_{a}\right)<0 .
$$

Corresponding to (3.12), we have

$$
g_{i}\left(E_{a}\right)=g_{i}\left(E_{1}\right)+g_{i}^{(E)}\left(E_{a}\right), \quad g_{i}^{(E)}\left(E_{1}\right)=0 .
$$

It is easily shown from (4.1) and (4.2) that

$$
\begin{gathered}
G_{+}^{\prime}\left(\omega ; E_{a}\right)=\sum_{i=1}^{n} \frac{g_{i}\left(E_{a}\right)}{\alpha_{i}+i \omega}, \\
G_{c}^{\prime}\left(\omega ; E_{a}\right)=\sum_{i=1}^{n} \frac{\alpha_{i} g_{i}\left(E_{a}\right)}{\alpha_{i}^{2}+\omega^{2}}, \quad G_{s}^{\prime}\left(\omega ; E_{a}\right)=\omega \sum_{i=1}^{n} \frac{g_{i}\left(E_{a}\right)}{\alpha_{i}^{2}+\omega^{2}} .
\end{gathered}
$$

Thus,

$$
\begin{aligned}
H\left(\omega ; E_{a}\right) & =-\omega^{2} \sum_{i=1}^{n} \frac{g_{i}\left(E_{a}\right)}{\alpha_{i}^{2}+\omega^{2}} \\
& =H_{\infty}\left(E_{a}\right)+\sum_{i=1}^{n} \frac{\alpha_{i}^{2} g_{i}\left(E_{a}\right)}{\alpha_{i}^{2}+\omega^{2}} \geq 0, \\
H_{\infty}\left(E_{a}\right) & =H\left(\infty ; E_{a}\right)=-\sum_{i=1}^{n} g_{i}\left(E_{a}\right) .
\end{aligned}
$$

Observe that $f(z)=H\left(\omega ; E_{a}\right), z=-\omega^{2}$, has simple poles at $\alpha_{i}^{2}, i=1,2, \ldots, n$. It will therefore have zeros at $\gamma_{i}^{2}\left(E_{a}\right), i=2,3, \ldots, n$, where

$$
\alpha_{1}^{2}<\gamma_{2}^{2}\left(E_{a}\right)<\alpha_{2}^{2}<\gamma_{3}^{2}\left(E_{a}\right) \ldots
$$

The function $f(z)$ also vanishes at $\gamma_{1}=0$. Therefore, $H$ is a rational function of the form

$$
H\left(\omega ; E_{a}\right)=H_{\infty}\left(E_{a}\right) \prod_{i=1}^{n}\left\{\frac{\gamma_{i}^{2}\left(E_{a}\right)+\omega^{2}}{\alpha_{i}^{2}+\omega^{2}}\right\}
$$


It follows from (6.7) and (4.9) that

$$
\begin{aligned}
H_{+}\left(\omega ; E_{a}\right) & =h_{\infty}\left(E_{a}\right) \prod_{i=1}^{n}\left\{\frac{\omega-i \gamma_{i}\left(E_{a}\right)}{\omega-i \alpha_{i}}\right\}, \\
H_{-}\left(\omega ; E_{a}\right) & =h_{\infty}\left(E_{a}\right) \prod_{i=1}^{n}\left\{\frac{\omega+i \gamma_{i}\left(E_{a}\right)}{\omega+i \alpha_{i}}\right\}, \\
h_{\infty}\left(E_{a}\right) & =\left[H_{\infty}\left(E_{a}\right)\right]^{1 / 2} .
\end{aligned}
$$

By considering the residue at each pole, we find that

$$
\begin{aligned}
& H_{-}\left(\omega ; E_{a}\right)=h_{\infty}\left(E_{a}\right)\left[1+i \sum_{i=1}^{n} \frac{R_{i}\left(E_{a}\right)}{\omega+i \alpha_{i}}\right], \\
& H_{+}\left(\omega ; E_{a}\right)=\overline{H_{-}}\left(\omega ; E_{a}\right), \\
& R_{i}\left(E_{a}\right)=\left(\gamma_{i}\left(E_{a}\right)-\alpha_{i}\right) \prod_{\substack{j=1 \\
j \neq i}}^{n}\left\{\frac{\gamma_{j}\left(E_{a}\right)-\alpha_{i}}{\alpha_{j}-\alpha_{i}}\right\} .
\end{aligned}
$$

Note that, using (6.7), we can write $H\left(\omega ; E_{a}\right)$ in the form

$$
\begin{aligned}
& H\left(\omega ; E_{a}\right)=H_{\infty}\left(E_{a}\right) P\left(\omega ; E_{a}\right) \prod_{i=1}^{n}\left\{\frac{1}{\left(\omega-i \alpha_{i}\right)\left(\omega+i \alpha_{i}\right)}\right\}, \\
& P\left(\omega ; E_{a}\right)=\prod_{i=1}^{n}\left(\omega^{2}+\gamma_{i}^{2}\left(E_{a}\right)\right) .
\end{aligned}
$$

We now show that minimal states for discrete spectrum materials can be characterized in a simple manner, generalizing the corresponding result in the linear case. From (5.10) and (6.2), we see that

$$
I_{d}^{t}(y ; E(t+s))=\sum_{i=1}^{n} g_{i}(E(t+s)) E_{d+}^{t}\left(-i \alpha_{i}\right) e^{-\alpha_{i} y} \quad \forall y, s \in \mathbb{R}^{+},
$$

where $E_{d+}^{t}\left(-i \alpha_{i}\right)$ is given by (5.4) and (4.15) for $\omega=-i \alpha_{i}$, so that they are real. Therefore, (5.17) 1 is obeyed if and only if

$$
E_{d}^{t}\left(-i \alpha_{i}\right)=E_{+1}^{t}\left(-i \alpha_{i}\right)-E_{+2}^{t}\left(-i \alpha_{i}\right)=0, \quad i=1,2, \ldots, n .
$$

This is the same characterization that applies in the linear case (2, page 362).

Using (6.5) 1 , we can write $M^{t}(E(t+s))$ and $M_{E}^{t}(E(t+s))$, given by (5.19), in the form

$$
\begin{aligned}
M^{t}(E(t+s)) & =-\sum_{i=1}^{n} g_{i}(E(t+s)) \Gamma^{t}\left(\alpha_{i}\right) \quad \forall s \in \mathbb{R}^{+}, \\
M_{E}^{t}(E(t+s)) & =-\sum_{i=1}^{n} g_{i E}(E(t+s)) \Gamma^{t}\left(\alpha_{i}\right) \quad \forall s \in \mathbb{R}^{+}, \\
\Gamma^{t}(\alpha) & =\frac{1}{2 \pi} \int_{-\infty}^{\infty} \frac{\omega^{2}}{\omega^{2}+\alpha^{2}}\left|E^{t}(\omega)\right|^{2} d \omega
\end{aligned}
$$


The quantities $g_{i E}(E(t+s)), i=1,2, \ldots, n$, vary independently of each other as functions of $s$ (except perhaps for some degenerate cases), and we must have

$$
\Gamma_{1}^{t}\left(\alpha_{i}\right)=\Gamma_{2}^{t}\left(\alpha_{i}\right), \quad i=1,2, \ldots, n
$$

which are quadratic conditions in the histories. The constraints (6.12) must be supplemented by these additional requirements.

Therefore, by virtue of (5.17) 2 , the set of histories in a given minimal state is in general a proper subset of the equivalent set for the linear problem.

Also, the existence of any branch cuts in $G_{+}^{\prime}(\omega ; E(t+s))$ will be sufficient to ensure that the minimal state is a singleton, by the same argument as in the linear case ([2], page 341), since (5.17) 1 is essentially the condition for equivalence in relation to linear memory materials.

Let us denote by $\sigma\left(t ; E_{1}\right)$ the quantity $\sigma(t)$, defined by $(3.8)_{2}$, where $E(t)$ is replaced by $E_{1}$ only in the kernels, so that

$$
\sigma\left(t ; E_{1}\right)=\phi(t)-\frac{1}{2}\left[G_{\infty}\left(E_{1}\right)-G_{0}\left(E_{1}\right)\right] E^{2}(t)+E(t) \int_{0}^{\infty} G^{\prime}\left(u ; E_{1}\right) E^{t}(u) d u .
$$

Then, from (4.19) $2,4.204,45.19)_{1},(6.3)$ and $(6.13)$, the work function terms in (3.26) can be written as

$$
\begin{aligned}
W(t ; E(t))-W\left(t ; E_{1}\right)=\sigma(t) & -\sigma\left(t, E_{1}\right)+M^{t}(E(t))-M^{t}\left(E_{1}\right) \\
=-\frac{1}{2}\left\{\left[G_{\infty}(E(t))-G_{\infty}\left(E_{1}\right)\right]\right. & \left.-\left[G_{0}(E(t))-G_{0}\left(E_{1}\right)\right]\right\} E^{2}(t) \\
& +E(t) \sum_{i=1}^{n} g_{i}^{(E)}(E(t)) E_{+}^{t}\left(-i \alpha_{i}\right) e^{-\alpha_{i} y} \\
& -\sum_{i=1}^{n} g_{i}^{(E)}(E(t)) \Gamma^{t}\left(\alpha_{i}\right) .
\end{aligned}
$$

Relations (6.12) and (6.14), combined with (6.16), yield that the left-hand side of (6.16) is a functional of the minimal state. This confirms the general result given by Proposition 5.2

A more detailed approach can be adopted to the issue of determining the nature of the minimal state for discrete spectrum materials in relation to that for the linear memory problem.

As before, we take $E_{1}^{t}$ to be a given history, and the minimal state is defined by the set of histories $E_{2}^{t}$ obeying (5.1). Let us put

$$
E_{2}^{t}(u)=E_{1}^{t}(u)+E_{d+}^{t}(u) .
$$

At large $\omega$, we must have

$$
E_{d+}^{t}(\omega) \sim \frac{1}{\omega^{2}},
$$


since $E_{1}(t)=E_{2}(t)$ (see (4.18) $)$. For linear memory discrete spectrum materials [15], the frequency domain representation of $E_{d}^{t}$ has the form

$$
E_{d+}^{t}(\omega)=\frac{E_{0}(\omega, t)}{\omega-i \chi_{0}} \prod_{j=1}^{n}\left\{\frac{\omega+i \alpha_{j}}{\omega-i \chi_{j}}\right\} \frac{1}{\omega-i \chi_{n+1}},
$$

where the constants $\chi_{i}, i=0,1, \ldots, n+1$, indicate the positions of singularities on the imaginary axis in $\Omega^{(+)}$. These are arbitrary positive quantities. The quantity $E_{0}(\omega, t)$ has the property that $\lim _{\omega \rightarrow \infty} E_{0}(\omega, t)$ is a non-zero finite constant, and the singularities of this quantity, if any, are branch cuts in $\Omega^{(+)}$.

Using (6.17) in (5.20), we obtain

$$
\begin{aligned}
\int_{-\infty}^{\infty} & \overline{E_{d+}^{t}}(\omega) H_{E}(\omega ; E(t+s)) E_{1+}^{t}(\omega) d \omega \\
& +\int_{-\infty}^{\infty} \overline{E_{1+}^{t}}(\omega) H_{E}(\omega ; E(t+s)) E_{d+}^{t}(\omega) d \omega \\
& +\int_{-\infty}^{\infty} \overline{E_{d+}^{t}}(\omega) H_{E}(\omega ; E(t+s)) E_{d+}^{t}(\omega) d \omega=0 .
\end{aligned}
$$

Substituting from (6.10) and (6.19), this condition gives

$$
\begin{aligned}
& \int_{-\infty}^{\infty} \overline{E_{0}}(\omega, t) \prod_{i=0}^{n+1}\left\{\frac{1}{\omega+i \chi_{i}}\right\} P_{E}(\omega ; E(t+s)) \prod_{i=1}^{n}\left\{\frac{1}{\omega+i \alpha_{i}}\right\} E_{1+}^{t}(\omega) d \omega \\
& +\int_{-\infty}^{\infty} \overline{E_{1+}^{t}}(\omega) P_{E}(\omega ; E(t+s)) \prod_{i=1}^{n}\left\{\frac{1}{\omega-i \alpha_{i}}\right\} E_{0}(\omega, t) \prod_{i=0}^{n+1}\left\{\frac{1}{\omega-i \chi_{i}}\right\} d \omega \\
& +\int_{-\infty}^{\infty} P_{E}(\omega ; E(t+s))\left|E_{0}(\omega, t)\right|^{2} \prod_{i=0}^{n+1}\left\{\frac{1}{\omega^{2}+\chi_{i}^{2}}\right\} d \omega=0, \quad s \geq 0 .
\end{aligned}
$$

The left-hand side of this equation is a function of $\lambda_{0}, \lambda_{1}, \lambda_{2}, \ldots, \lambda_{n+1}$, and the condition reduces the number of free parameters by one, if solutions exist. Indeed, if we expand $P_{E}\left(w ; E_{a}\right)$ in terms of the quantities $g_{i}(E(t+s))$, which may be regarded as independent functions of $s$, similarly to the situation in (6.13), we must write $n$ separate conditions on the $\lambda_{i}, i=0,1, \ldots, n+1$. This reduces the number of independent parameters to two if meaningful solutions to the $n$ conditions exist. Thus, the size of the minimal state is greatly reduced by $(5.17)_{2}$.

7. Generalization of some standard free energy functionals. It is of interest to determine what are the natural generalizations of standard free energy functional forms in the linear memory case to the non-linear materials of interest here. Notably, there are the Graffi-Volterra, the Dill and the Fabrizio functionals which are free energies in the linear memory case, provided the relaxation function obeys certain decay conditions ([2], pages 217,219 and 221). 
We will first discuss the Graffi-Volterra case, for which the free energy functional is denoted by $\psi_{G V}$. Following the prescription given by (3.26), we put

$$
\psi_{G V}(t)=\phi(t)-\frac{1}{2} \int_{0}^{\infty} G^{\prime}\left(u ; E_{1}\right)\left[E_{r}^{t}(u)\right]^{2} d u+W(t ; E(t))-W\left(t ; E_{1}\right),
$$

where $G^{\prime}\left(u ; E_{a}\right)$ for $E_{a}=E(t)$ or $E_{1}$ is the specified relaxation function for the material, to be used in the first integral term and also in the second and third terms, in accordance with (3.14) and (3.17) 3 . It must obey the constraints

$$
G^{\prime}\left(u ; E_{a}\right) \leq 0, \quad G^{\prime \prime}\left(u ; E_{a}\right) \geq 0, \quad E_{a}=E(t) \text { or } E_{1} .
$$

There may be a separate free energy for every choice of $E_{1}$. An example of $G^{\prime}\left(u ; E_{1}\right)$ is given by (3.13) if $G^{(i)}(u), i=0,2$, both obey the conditions (7.23).

Note that it would not be correct to take $\psi_{G V}(t)$ as simply the first two terms of (7.22) with $E_{1}$ replaced by $E(t)$, though this may seem to be a natural choice. However, it does not give the correct quadratic term in the constitutive relation, as specified by (3.30). A similar observation applies to the two other cases now discussed.

The generalization of the Fabrizio free energy is given by essentially the same argument to be

$$
\psi_{F}(t)=\phi(t)-\frac{1}{2} \int_{0}^{\infty} \frac{\left[I_{(1)}^{t}\left(u ; E_{1}\right)\right]^{2}}{G^{\prime}\left(u ; E_{1}\right)} d u+W(t ; E(t))-W\left(t ; E_{1}\right),
$$

also subject to the constraints (7.23). The quantity $I_{(1)}^{t}\left(u ; E_{1}\right)$ is defined by (5.11) 2 .

Similarly, the generalization of the Dill free energy is given by

$$
\begin{aligned}
\psi_{\text {Dill }}(t)=\phi(t) & +\frac{1}{2} \int_{0}^{\infty} G^{\prime \prime}\left(u+v ; E_{1}\right) E_{r}^{t}(u) E_{r}^{t}(v) d u d v \\
& +W(t ; E(t))-W\left(t ; E_{1}\right),
\end{aligned}
$$

subject to a stronger constraint than (7.23), namely that $G$ be completely monotonic. This requires that relations such as (7.23) apply also to all higher derivatives (2, page 219).

The rates of dissipation for these functionals may be determined by $(3.29)_{2}$. In each case, these are given by the linear memory formula, using the relaxation function at $E_{1}$, as given in 2, pages 218, 219 and 223 .

It follows from Proposition 5.2 that $\psi_{F}(t)$ and $\psi_{\text {Dill }}(t)$ are functionals of the minimal state. The Graffi-Volterra functional does not have this property.

8. The minimum and related free energies. Our object now is to deduce the form of the minimum free energy $\psi_{m}(t)$ from among all the possibilities for $\psi(t)$, given by (3.26). These various choices relate only to the linear memory free energy $\psi_{l}(t)$. The minimum free energy for this linear memory problem, which we denote by $\psi_{l m}(t)$, is well-known and will be described below.

There is also a family of free energies related to $\psi_{l m}(t)$ for materials with only isolated singularities, in particular for discrete spectrum materials, as defined in section 6 . These are discussed in subsection 8.2 
8.1. The form of the minimum free energy. The quantity $H_{-}\left(\omega ; E_{1}\right) E_{r+}^{t}(\omega)$ is continuous, indeed analytic, on $\mathbb{R}$ by virtue of the analyticity properties of $H_{-}\left(\omega ; E_{1}\right)$ and $E_{+}^{t}(\omega)$. The Plemelj formulae ([2], page 542) give that

$$
H_{-}\left(\omega ; E_{1}\right) E_{r+}^{t}(\omega)=p_{-}^{t}\left(\omega ; E_{1}\right)-p_{+}^{t}\left(\omega ; E_{1}\right)
$$

where

$$
\begin{aligned}
p^{t}\left(z ; E_{1}\right) & =\frac{1}{2 \pi i} \int_{-\infty}^{\infty} \frac{H_{-}\left(\omega^{\prime} ; E_{1}\right) E_{r+}^{t}\left(\omega^{\prime}\right)}{\omega^{\prime}-z} d \omega^{\prime}, \\
p_{ \pm}^{t}\left(\omega ; E_{1}\right) & =\lim _{\alpha \rightarrow 0 \mp} p^{t}\left(\omega+i \alpha ; E_{1}\right) .
\end{aligned}
$$

Moreover, $p^{t}\left(z ; E_{1}\right)=p_{+}^{t}\left(z ; E_{1}\right)$ is analytic in $z \in \Omega^{(-)}$, and $p^{t}\left(z ; E_{1}\right)=p_{-}^{t}\left(z ; E_{1}\right)$ is analytic in $z \in \Omega^{(+)}$. Both are analytic on the real axis. We write them in the form

$$
p_{ \pm}^{t}\left(\omega ; E_{1}\right)=\frac{1}{2 \pi i} \int_{-\infty}^{\infty} \frac{H_{-}\left(\omega^{\prime} ; E_{1}\right) E_{r+}^{t}\left(\omega^{\prime}\right)}{\omega^{\prime}-\omega^{\mp}} d \omega^{\prime},
$$

where the notation $\omega^{ \pm}$is that introduced in and after (4.17). Observe that in $p_{ \pm}^{t}\left(\omega ; E_{1}\right)$, the fixed strain $E_{1}$ occurs in $H_{-}(\omega ; \cdot)$ but not in $E_{r+}^{t}(\omega)$, which is given by (4.17). The quantity $p_{+}^{t}\left(\omega ; E_{1}\right)$ is independent of $E(t)$. Indeed, one can show that

$$
p_{+}^{t}\left(\omega ; E_{1}\right)=\frac{1}{2 \pi i} \int_{-\infty}^{\infty} \frac{H_{-}\left(\omega^{\prime} ; E_{1}\right) E_{+}^{t}\left(\omega^{\prime}\right)}{\omega^{\prime}-\omega^{-}} d \omega^{\prime},
$$

using the analytic behavior of $H_{-}\left(\omega ; E_{1}\right)$.

Let us define $p_{ \pm}^{t}(\omega ; E(t))$ as the quantities given by (8.3) but with $E_{1}$ replaced by $E(t)$. Then, from (4.19), 4.9) and (8.1),

$$
\begin{aligned}
W(t) & =W(t ; E(t))=\phi(t)+\frac{1}{2 \pi} \int_{-\infty}^{\infty}\left|p_{-}^{t}(\omega ; E(t))-p_{+}^{t}(\omega ; E(t))\right|^{2} d \omega \\
& =\phi(t)+\frac{1}{2 \pi} \int_{-\infty}^{\infty}\left[\left|p_{-}^{t}(\omega ; E(t))\right|^{2}+\left|p_{+}^{t}(\omega ; E(t))\right|^{2}\right] d \omega,
\end{aligned}
$$

since the cross-terms vanish ([2], page 538). Also,

$$
W\left(t ; E_{1}\right)=\phi(t)+\frac{1}{2 \pi} \int_{-\infty}^{\infty}\left[\left|p_{-}^{t}\left(\omega ; E_{1}\right)\right|^{2}+\left|p_{+}^{t}\left(\omega ; E_{1}\right)\right|^{2}\right] d \omega .
$$

Replacing $\psi_{l}\left(t ; E_{1}\right)$ in $(3.26)$ by the expression for the minimum free energy (see for example 2, page 243), we obtain, for a given choice of $E_{1}$,

$$
\begin{aligned}
\psi_{m l}\left(t ; E_{1}\right) & =\phi(t)+\frac{1}{2 \pi} \int_{-\infty}^{\infty}\left[\left|p_{-}^{t}\left(\omega ; E_{1}\right)\right|^{2} d \omega\right. \\
\psi_{m}\left(t ; E_{1}\right) & =\psi_{m l}\left(t ; E_{1}\right)+W(t ; E(t))-W\left(t ; E_{1}\right) \\
& =W(t ; E(t))-\frac{1}{2 \pi} \int_{-\infty}^{\infty}\left|p_{+}^{t}\left(\omega ; E_{1}\right)\right|^{2} d \omega \\
& =\phi(t)+\frac{1}{2 \pi} \int_{-\infty}^{\infty}\left[\left|p_{-}^{t}(\omega ; E(t))\right|^{2}+\left|p_{+}^{t}(\omega ; E(t))\right|^{2}\right. \\
& \left.-\left|p_{+}^{t}\left(\omega ; E_{1}\right)\right|^{2}\right] d \omega .
\end{aligned}
$$

For the linear memory problem, the last two terms on the right of (8.7) 4 cancel, and we are left with the first two terms. 
Equation (2.9), in the case of the minimum free energy, takes the form

$$
\dot{\psi}_{m}\left(t ; E_{1}\right)+D_{m}\left(t ; E_{1}\right)=S(t) \dot{E}(t),
$$

where $D_{m}$ is the rate of dissipation corresponding to that free energy and must be nonnegative by the second law. Integrating (8.8) up to time $t$ gives

$$
\psi_{m}\left(t ; E_{1}\right)+\mathcal{D}_{m}\left(t ; E_{1}\right)=W(t ; E(t)),
$$

where

$$
\mathcal{D}_{m}\left(t ; E_{1}\right)=\int_{-\infty}^{t} D_{m}\left(s ; E_{1}\right) d s
$$

is the total dissipation up to time $t$, corresponding to the minimum free energy. These are special cases of (2.10) and (2.11) 2 . Since $\psi_{m}\left(t ; E_{1}\right)$ is less than or equal to any other free energy, for a specific choice $E_{1}$, it follows from $(8.9)$ that $\mathcal{D}_{m}\left(t ; E_{1}\right)$ is the largest estimate of dissipation in the material element. We have, from $(8.7)_{3}$,

$$
\begin{aligned}
\mathcal{D}_{m}\left(t ; E_{1}\right) & =W(t ; E(t))-\psi_{m}\left(t ; E_{1}\right) \\
& =W\left(t ; E_{1}\right)-\psi_{m l}\left(t ; E_{1}\right)=\frac{1}{2 \pi} \int_{-\infty}^{\infty}\left|p_{+}^{t}\left(\omega ; E_{1}\right)\right|^{2} d \omega \geq 0 .
\end{aligned}
$$

Relations (2.18) and (2.19) are obeyed for this form, by virtue of (8.4).

REMARK 8.1. The arbitrary quantity $E_{1}$ is fixed at a value $E_{0}$, which provides an optimum choice of minimum free energy. Consider the set of values of $\mathcal{D}_{m}\left(t ; E_{1}\right)$ as a function of $E_{1}$. If $\mathcal{D}_{m}\left(t ; E_{1}\right)$ has an overall maximum at one or more finite values of $E_{1}$, internal to the set, we choose one of these values to be $E_{0}$. If $\mathcal{D}_{m}\left(t ; E_{1}\right)$ increases without limit with $\left|E_{1}\right|$, then we put $E_{0}=E_{l}$, the limiting strain introduced in Remark 3.3. It is understood that $\mathcal{D}_{m}\left(t ; E_{a}\right) \leq \mathcal{D}_{m}\left(t ; E_{l}\right)$, for any $E_{a}$ in the theory.

Then, the overall minimum free energy is given by

$$
\psi_{m}(t)=\psi_{m}\left(t ; E_{0}\right), \quad \mathcal{D}_{m}(t)=\mathcal{D}_{m}\left(t ; E_{0}\right) .
$$

An explicit expression for $D_{m}(t)=\dot{\mathcal{D}}_{m}(t)$ follows by an identical argument to that given in 2], page 244. We find that

$$
D_{m}(t)=|K(t)|^{2}, \quad K(t)=\frac{1}{2 \pi} \int_{-\infty}^{\infty} H_{-}\left(\omega ; E_{0}\right) E_{r+}^{t}(\omega) d \omega .
$$

Now, $\psi_{m}\left(t ; E_{1}\right)$ has the characteristic properties P1 - P3 (or (2.7) - (2.9)) of a free energy listed in subsection 2.1. since this has been shown for the general form (3.26).

For the order two model, defined by (3.7) and (3.13) with $E_{c}=0$, we see from (4.11) and (8.3) that

$$
p_{+}^{t}\left(\omega ; E_{1}\right)=A(\omega)+B(\omega) E_{1},
$$

where

$$
\begin{aligned}
& A(\omega)=\frac{1}{2 \pi i} \int_{-\infty}^{\infty} \frac{H_{-}^{(0)}\left(\omega^{\prime}\right) E_{r+}^{t}\left(\omega^{\prime}\right)}{\omega^{\prime}-\omega^{-}} d \omega^{\prime}, \\
& B(\omega)=\frac{1}{2 \pi i} \int_{-\infty}^{\infty} \frac{H_{-}^{(2)}\left(\omega^{\prime}\right) E_{r+}^{t}\left(\omega^{\prime}\right)}{\omega^{\prime}-\omega^{-}} d \omega^{\prime} .
\end{aligned}
$$


Therefore

$$
\begin{aligned}
\mathcal{D}_{m}\left(t ; E_{1}\right) & =\frac{1}{2 \pi} \int_{-\infty}^{\infty}\left|A(\omega)+B(\omega) E_{1}\right|^{2} d \omega=C+D E_{1}+F E_{1}^{2}, \\
C & =\frac{1}{2 \pi} \int_{-\infty}^{\infty}|A(w)|^{2} d \omega, \\
D & =\frac{1}{2 \pi} \int_{-\infty}^{\infty}[A(w) \bar{B}(\omega)+B(w) \bar{A}(\omega)] d \omega, \\
F & =\frac{1}{2 \pi} \int_{-\infty}^{\infty}|B(w)|^{2} d \omega .
\end{aligned}
$$

This function increases without limit with $E_{1}^{2}$. Thus, we take $E_{0}=E_{l}$, as indicated in Remark 3.3.

REMARK 8.2. By virtue of Proposition 5.2 and the fact that $\psi_{m l}\left(t ; E_{1}\right)$ is a functional of the minimal state ([2], page 252), we conclude that $\psi_{m}\left(t ; E_{1}\right)$ has this property for all values of $E_{1}$, in particular $E_{0}$.

8.2. A family of free energies for materials with only isolated singularities. Consider materials such that the singularities of $H\left(\omega ; E_{1}\right)$ on the complex $\omega$ plane are all isolated, in the sense that no branch cuts exist. The special case of discrete spectrum materials, described in section [6] provide the simplest example. For such cases, a family of free energies can be defined (and ordered) as described in chapters 15, 16 of [2]. We label members of this family as $\psi_{f}\left(t ; E_{1}\right)$, where the subscript distinguishes between them. In particular, for $f=1$, we have $\psi_{f}\left(t ; E_{1}\right)=\psi_{m}\left(t ; E_{1}\right)$, the minimum free energy, while $f=N$ corresponds to the maximum free energy which is a functional of the minimal state, so that $\psi_{N}\left(t ; E_{1}\right)=\psi_{M}\left(t ; E_{1}\right)$, where the integer $N$ is defined in 2, page 342 .

The subscript $f$ indicates some interchanges of the zeros of $H_{ \pm}\left(\omega ; E_{1}\right)$ to obtain $H_{ \pm}^{f}\left(\omega ; E_{1}\right)$. The quantities $p_{ \pm}^{(f t)}\left(\omega ; E_{1}\right)$ are defined in terms of these factors by formulae analogous to (8.1) and (8.3). Then, corresponding to (8.7), for $E_{1}=E_{0}$, we have

$$
\psi_{f}\left(t ; E_{0}\right)=\phi(t)+\frac{1}{2 \pi} \int_{-\infty}^{\infty}\left[\left|p_{-}^{f t}\left(\omega ; E_{0}\right)\right|^{2} d \omega+W(t ; E(t))-W\left(t ; E_{0}\right) .\right.
$$

Also, corresponding to (8.13),

$$
\begin{aligned}
D_{f}\left(t ; E_{0}\right) & =\left|K_{f}(t)\right|^{2}, \\
K_{f}(t) & =\frac{1}{2 \pi} \int_{-\infty}^{\infty} H_{-}^{f}\left(\omega ; E_{0}\right) E_{r+}^{t}(\omega) d \omega .
\end{aligned}
$$

It can be shown that the quantities $\psi_{f}\left(t ; E_{0}\right)$ are functionals of the minimal state, in other words, obey (5.27), using the argument outlined in Remark 8.2 see also [2], page 352.

Analogous formulae apply to the proposed physical free energy functional (13, 14, [2], page 367).

9. Explicit forms of the minimum free energy for discrete spectrum materials. We now consider the general results of section 8 for discrete spectrum scalar models discussed in section 6. The formulae presented are simple generalizations of 
certain relations in [12] or [2], page 265. We shall evaluate the relevant quantities at $E_{a}=E_{0}$.

The quantity $p_{-}^{t}\left(\omega ; E_{0}\right)$ may be determined, using (6.9), by closing on $\Omega^{(-)}$to obtain

$$
p_{-}^{t}\left(\omega ; E_{0}\right)=i h_{\infty}\left(E_{0}\right) \sum_{i=1}^{n} \frac{R_{i}\left(E_{0}\right) E_{r+}^{t}\left(-i \alpha_{i}\right)}{\omega+i \alpha_{i}} .
$$

Also

$$
\begin{aligned}
p_{+}^{t}\left(\omega ; E_{0}\right)= & p_{-}^{t}\left(\omega ; E_{0}\right)-H_{-}\left(\omega ; E_{0}\right) E_{r+}^{t}(\omega) \\
= & i h_{\infty}\left(E_{0}\right) \sum_{i=1}^{n} R_{i}\left(E_{0}\right) \frac{\left[E_{r+}^{t}\left(-i \alpha_{i}\right)-E_{r+}^{t}(\omega)\right]}{\omega+i \alpha_{i}} \\
& \quad-h_{\infty}\left(E_{0}\right) E_{r+}^{t}(\omega),
\end{aligned}
$$

which has singularities at those of $E_{r+}^{t}(\omega)$ in $\Omega^{(+)}$but none in $\Omega^{(-)}$. These explicit relations for $p_{ \pm}^{t}$ allow their analytic continuation to the whole complex plane, excluding singular points.

We deduce from (8.7) and (9.1) that

$$
\begin{aligned}
\psi_{m}(t)=\phi(t) & +H_{\infty}\left(E_{0}\right) \sum_{i, j=1}^{n} \frac{R_{i}\left(E_{0}\right) R_{j}\left(E_{0}\right)}{\alpha_{i}+\alpha_{j}} E_{r+}^{t}\left(-i \alpha_{i}\right) E_{r+}^{t}\left(-i \alpha_{j}\right) \\
& +W(t ; E(t))-W\left(t ; E_{0}\right) \\
=\phi(t) & +\frac{1}{2} \int_{0}^{\infty} d s_{1} \int_{0}^{\infty} d s_{2} E_{r}^{t}\left(s_{1}\right) G_{12}\left(s_{1}, s_{2} ; E_{0}\right) E_{r}^{t}\left(s_{2}\right) \\
& +W(t ; E(t))-W\left(t ; E_{0}\right),
\end{aligned}
$$

where the kernel $G_{12}$ is given by

$$
G_{12}\left(s_{1}, s_{2} ; E_{0}\right)=2 H_{\infty}\left(E_{0}\right) \sum_{i, j=1}^{n} \frac{R_{i}\left(E_{0}\right) R_{j}\left(E_{0}\right)}{\alpha_{i}+\alpha_{j}} e^{-\alpha_{i} s_{1}-\alpha_{j} s_{2}} .
$$

From $(8.13) 2$ and $(6.9)$ we have

$$
K(t)=h_{\infty}\left(E_{0}\right) \sum_{i=1}^{n} R_{i}\left(E_{0}\right) E_{r+}^{t}\left(-i \alpha_{i}\right)
$$

since the constant term in $H_{-}\left(\omega ; E_{0}\right)$ yields zero. Then, from 8.13$)_{1}$,

$$
\begin{aligned}
D_{m}(t) & =H_{\infty}\left(E_{0}\right)\left[\sum_{i=1}^{n} R_{i}\left(E_{0}\right) E_{r+}^{t}\left(-i \alpha_{i}\right)\right]^{2} \\
& =H_{\infty}\left(E_{0}\right)\left[\int_{0}^{\infty} \sum_{i=1}^{n} R_{i}\left(E_{0}\right) e^{-\alpha_{i} s} E_{r}^{t}(s) d s\right]^{2} .
\end{aligned}
$$

The family of free energies discussed in subsection 8.2 is particularly simply described for discrete spectrum materials ([2, page 365). The quantities $\psi_{f}\left(t ; E_{1}\right)$ and $D_{f}\left(t ; E_{1}\right)$ are given by straightforward modifications of the above formulae, where the zeros $\gamma_{i}$ are replaced by $\rho_{i}^{f}$ given by

$$
\rho_{i}^{f}=\epsilon_{i}^{f} \gamma_{i}, \quad \epsilon_{i}^{f}= \pm 1, i=1,2 \ldots, n .
$$


Thus, $\epsilon_{i}^{f}= \pm 1, i=1,2 \ldots, n$, characterize the particular exchanges of zeros denoted by $f$.

\section{RefERENCES}

[1] Giovambattista Amendola, Mauro Fabrizio, and John Murrough Golden, Free energies in a general non-local theory of a material with memory, Math. Models Methods Appl. Sci. 24 (2014), no. 6, 1037-1090, DOI 10.1142/S0218202513500760. MR 3187191

[2] Giovambattista Amendola, Mauro Fabrizio, and John Murrough Golden, Thermodynamics of materials with memory, Theory and applications, Springer, New York, 2012. MR2856615(2012j:74001)

[3] Bernard D. Coleman, Thermodynamics of materials with memory, Arch. Rational Mech. Anal. 17 (1964), 1-46. MR0171419(30 \#1650)

[4] Bernard D. Coleman and Walter Noll, Foundations of linear viscoelasticity, Rev. Modern Phys. 33 (1961), 239-249. MR0158605 (28 \#1828)

[5] Gianpietro Del Piero and Luca Deseri, On the analytic expression of the free energy in linear viscoelasticity, J. Elasticity 43 (1996), no. 3, 247-278, DOI 10.1007/BF00042503. MR1415545 (97g:73042)

[6] Gianpietro Del Piero and Luca Deseri, On the concepts of state and free energy in linear viscoelasticity, Arch. Rational Mech. Anal. 138 (1997), no. 1, 1-35, DOI 10.1007/s002050050035. MR 1463802 (98i:73023)

[7] Luca Deseri, Giorgio Gentili, and Murrough Golden, An explicit formula for the minimum free energy in linear viscoelasticity, J. Elasticity 54 (1999), no. 2, 141-185, DOI 10.1023/A:1007646017347. MR.1728444 (2001i:74012)

[8] Luca Deseri, Mauro Fabrizio, and Murrough Golden, The concept of minimal state in viscoelasticity: new free energies and applications to PDEs, Arch. Ration. Mech. Anal. 181 (2006), no. 1, 43-96, DOI 10.1007/s00205-005-0406-1. MR2221203 (2009a:74024)

[9] L. Deseri and J. M. Golden, The minimum free energy for continuous spectrum materials, SIAM J. Appl. Math. 67 (2007), no. 3, 869-892 (electronic), DOI 10.1137/050639776. MR2300315 (2008d:74003)

[10] Mauro Fabrizio and Angelo Morro, Mathematical problems in linear viscoelasticity, SIAM Studies in Applied Mathematics, vol. 12, Society for Industrial and Applied Mathematics (SIAM), Philadelphia, PA, 1992. MR1153021 (93a:73034)

[11] M. Fabrizio and J. M. Golden, Maximum and minimum free energies for a linear viscoelastic material, Quart. Appl. Math. 60 (2002), no. 2, 341-381. MR1900497(2003b:74013)

[12] J. M. Golden, Free energies in the frequency domain: the scalar case, Quart. Appl. Math. 58 (2000), no. 1, 127-150. MR1739041 (2001e:74025)

[13] J. M. Golden, A proposal concerning the physical rate of dissipation in materials with memory, Quart. Appl. Math. 63 (2005), no. 1, 117-155, DOI 10.1177/1081286506061450. MR2126572 (2005k:74002)

[14] J. M. Golden, A proposal concerning the physical rate of dissipation of materials with memory: the non-isothermal case, Math. Mech. Solids 12 (2007), no. 4, 403-449, DOI 10.1177/1081286505061450. MR2349161 (2008i:74008)

[15] J. M. Golden, Free energies for materials with memory in terms of state functionals, Meccanica 49 (2014), no. 9, 2207-2235, DOI 10.1007/s11012-014-9967-0. MR 3240290

[16] Dario Graffi and Mauro Fabrizio, On the notion of state for viscoelastic materials of "rate" type (Italian, with English summary), Atti Accad. Naz. Lincei Rend. Cl. Sci. Fis. Mat. Natur. (8) 83 (1989), 201-208 (1990). MR1142459 (93a:73036)

[17] Dario Graffi, More on the analytic expression of free energy in materials with memory (Italian), Writings in mathematical physics in honor of the ninetieth birthday of Cataldo Agostinelli (Italian), Atti Accad. Sci. Torino Cl. Sci. Fis. Mat. Natur. 120 (1986), suppl., 111-124 (1987). MR958166 (90f:73030)

[18] Walter Noll, A new mathematical theory of simple materials, Arch. Rational Mech. Anal. 48 (1972), 1-50. MR0445985(56 \#4318) 